\title{
Large-sized species of Ctenodactylidae from the Valley of Lakes (Mongolia): An update on dental morphology, biostratigraphy, and paleobiogeography
}

\author{
Adriana Oliver and Gudrun Daxner-Höck
}

\begin{abstract}
Our study of the large and medium-sized species of Yindirtemys from the Valley of Lakes in Mongolia yielded three species: Yindirtemys deflexus, Y. suni and Y. birgeri. They differ in size and dental morphology. Yindirtemys suni is the largest and Y. birgeri the smallest species. Yindirtemys deflexus and $Y$. suni increase in body size but do not change dental morphology within their time range. The medium-sized Yindirtemys birgeri co-occurs in Mongolia with Y. deflexus in the late Oligocene (biozone C1) and is the easternmost occurrence of the species so far. Hitherto, Y. birgeri has been recorded from the Aral region in Kazakhstan only. Both Yindirtemys deflexus and $Y$. suni are used as biostratigraphic markers. Yindirtemys deflexus is a "key fossil" of the Chinese Land Mammal Age Tabenbulukian and for the Mongolian biozone C1; the latter correlates with the Oligocene. Yindirtemys suni is one of the "key fossils" of the Chinese Land Mammal Age Xiejian. In Mongolia the species ranges from the OligoceneMiocene transition (biozone C1-D) to the earliest Miocene (biozone D). The wide geographic range of Yindirtemys in the Oligocene of Asia suggests that no major physical barriers hampered mammal distribution between Kazakhstan, Mongolia and northern China, but local paleoecological and climatic conditions might have influenced mammal community structures in these areas.
\end{abstract}

Adriana Oliver. Museo Nacional de Ciencias Naturales, MNCN-CSIC, C/ José Gutiérrez Abascal, 2, 28006, Madrid, Spain. Geological-Paleontological Department, Natural History Museum Vienna. Burgring 7, 1010 Vienna, Austria. adriana@mncn.csic.es

Gudrun Daxner-Höck. Geological-Paleontological Department, Natural History Museum Vienna. Burgring 7, 1010 Vienna, Austria.gudrun.hoeck@nhm-wien.ac.at

Keywords: Small mammals; Yindirtemys; Asia; Paleogene; Oligocene; Miocene

Submission: 15 February 2016 Acceptance: 22 December 2016

Oliver, Adriana and Daxner-Höck, Gudrun. 2017. Large-sized species of Ctenodactylidae from the Valley of Lakes (Mongolia): An update on dental morphology, biostratigraphy, and paleobiogeography. Palaeontologia Electronica 20.1.1A: 1-22 palaeo-electronica.org/content/2017/1729-ctenodactylidae-from-mongolia 


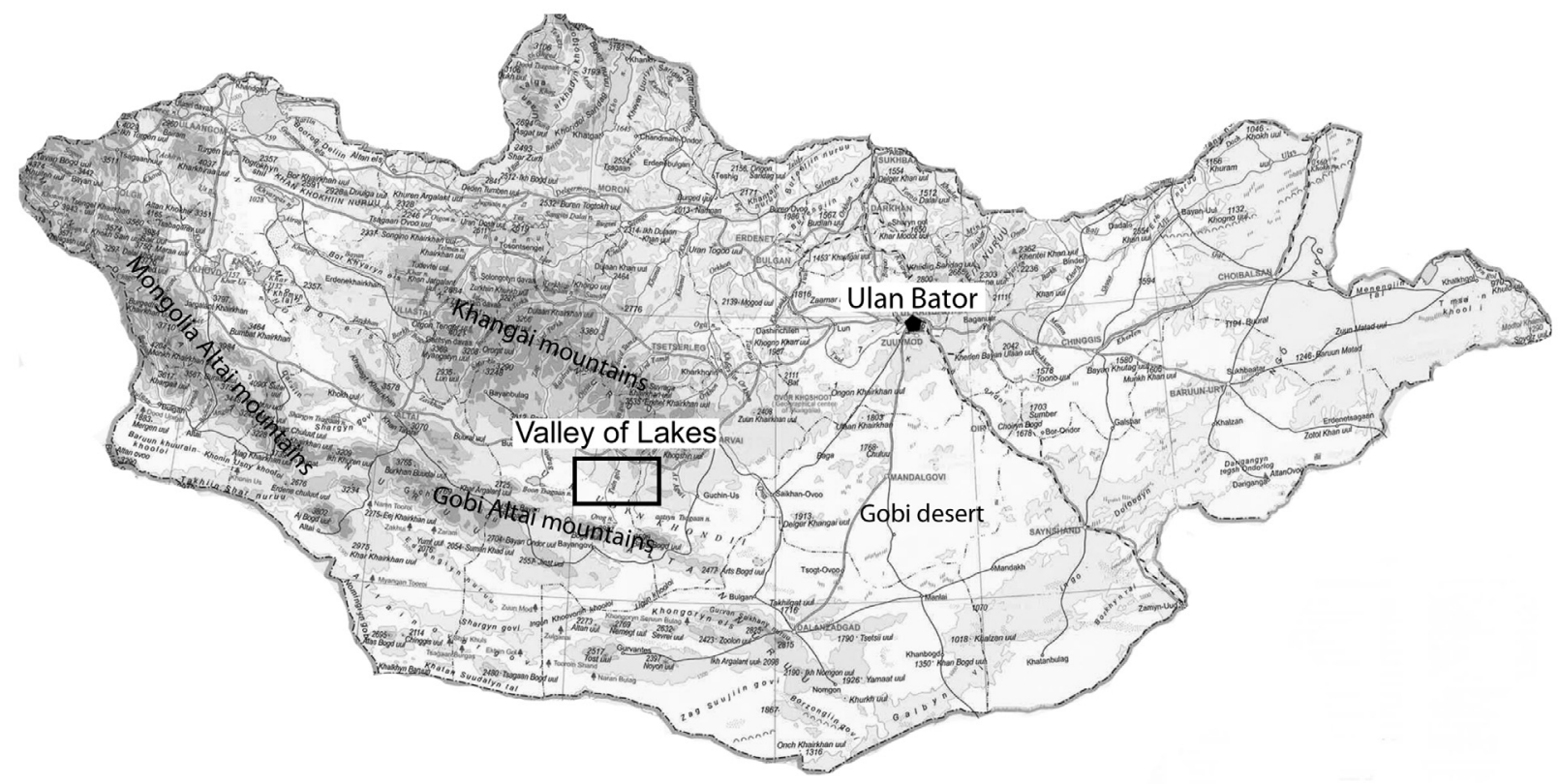

FIGURE 1. Map of Mongolia, showing the location of the working area in the Valley of Lakes (Modified from Map of Mongolia by Cartography Co. Ltd, Ulan Bator, Mongolia).

\section{INTRODUCTION}

The rodent family Ctenodactylidae is characterized by the combination of a hystricomorphous skull, a sciurognathous mandible, a well-developed lower masseteric crest, and incisor enamel with multiserial microstructure (Wang, 1997). This family has its first occurrence in Asia during the Eocene; it diversified and became one of the dominant rodent groups during the Oligocene. In the early Miocene, most members of the family became extinct (Wang, 1994). Only the subfamily Ctenodactylinae survived, diversified and spread into the Indian subcontinent, the Middle East, some Mediterranean islands and North Africa. Today, four genera and five species of the subfamily inhabit North Africa.

Ctenodactylids are excellent biostratigraphic markers and are useful tools for paleobiogeography and paleoenvironmental reconstructions (Bendukidze et al., 2009; Vianey-Liaud et al., 2010; Gomes Rodrigues et al., 2012); their fossil richness, high diversity and rapid evolution made them "key fossils" for biostratigraphy of the Paleogene in Asia (Bohlin, 1946; Kowalski, 1974; Li and Qiu, 1980; Huang, 1985; Wang, 1997; Höck et al., 1999; Vianey-Liaud et al., 2006; Daxner-Höck and Badamgarav, 2007; Daxner-Höck et al., 2010, 2015).

The present study is a revision and description of the larger-sized Yindirtemys species, which are characteristic of the Oligocene and early Miocene of Mongolia. It is based on new and already known fossil material from the Valley of Lakes (Mongolia) and describes the temporal and geographic distribution of Yindirtemys in Asia.

The study area is the Taatsiin Gol and Taatsiin Tsagaan Nuur region, a part of the Valley of Lakes in Central Mongolia (Figure 1). The fossils examined in this study were collected from 30 layers of 10 sections and localities. The excavations were conducted during eight field seasons within the Mongolian-Austrian joint projects between 1995 and 2012.

\section{MATERIAL AND METHODS}

We studied and redescribed the Ctenodactylidae material from the collections of the years 1995 to 1997 , previously described by Schmidt-Kittler et al. (2007), as well as new fossils collected between 2001 and 2011.

The dental terminology of Yindirtemys (Figure 2) is modified after Wang (1997) and Vianey-Liaud et al. (2006). Notation $M$ and $m$ is used for upper and lower molars, respectively. The photographs of the occlusal surface of cheek teeth were made with a Scanning Electron Microscope Philips XL 30 at the Core Facility of Cell Imaging and Ultrastructure Research (CIUS) EM LAB, Faculty of Life Sciences, University Vienna (Austria) and with a Scanning Electron Microscope Fei, model Quanta 

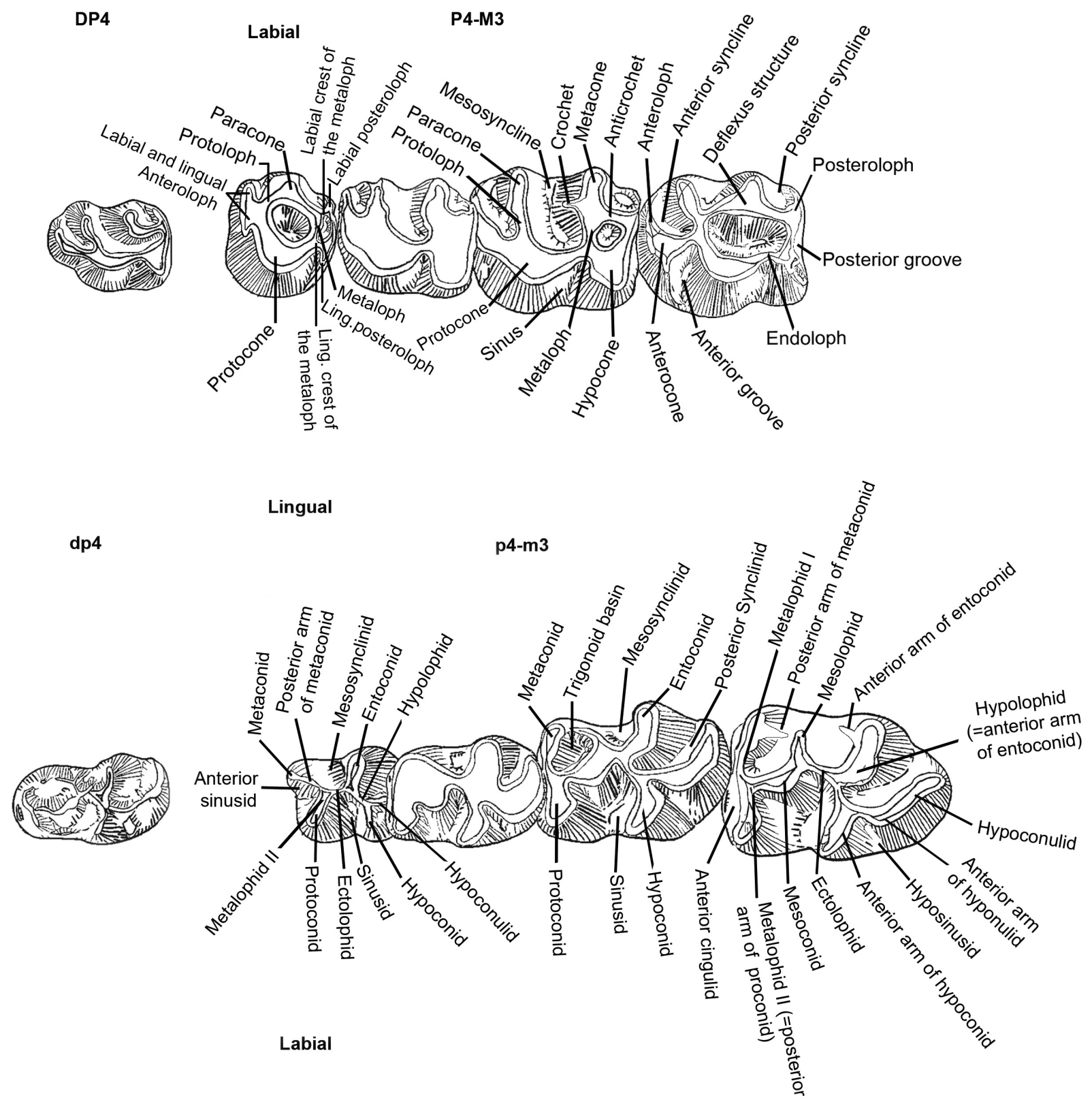

FIGURE 2. Dental terminology of the upper and lower cheek teeth of Yindirtemys deflexus. Terminology after VianeyLiaud et al. (2006) and Wang (1997). Illustration modified from Schmidt-Kittler et al. (2007).

200 at the National Museum of Natural SciencesSpanish National Research Council (Madrid, Spain). The morphological analyses are listed in comparative tables (Appendix 1 and 2, description of character states and distribution of character states). Abbreviations of mammal assemblages and section/fossil points are included in Table 1.

Measurements were taken using Discovery V20 and Carl Zeiss software Axiocam MRc5 and are given in millimeters. Length and width repre- sent the maximum antero-posterior and labio-lingual distances, respectively, taken perpendicular to each other.

The descriptive statistics were calculated in SPSS version 15.0.1 (SPSS, 2006) and included in Tables 2, 3, 4, and 5. To test significant differences among fossil assemblages, we carried out analysis of variance (One-Way ANOVA). Previous to the ANOVA, Levene's test was calculated to check for homogeneity of variance. In case of significant 
TABLE 1. Abbreviations of mammal assemblages including the biozone, the section/fossil point and the locality name.

\begin{tabular}{|c|c|c|c|}
\hline Assemblage/fossil layer & Biozone & Section/fossil point & Locality name \\
\hline $\mathrm{LOH}-\mathrm{C} / 1$ & C1 & $\mathrm{LOH}-\mathrm{C}$ & Loh \\
\hline $\mathrm{IKH}-\mathrm{A} / 5$ & $\mathrm{C} 1$ & $\mathrm{IKH}-\mathrm{A}$ & Ikh Argalatyn Nuru \\
\hline $\mathrm{IKH}-\mathrm{B} / 5$ & C1 & $\mathrm{IKH}-\mathrm{B}$ & Ikh Argalatyn Nuru \\
\hline DEL-B/12 & C1 & DEL-B & Del \\
\hline TGW-A/surface & $\mathrm{C} 1$ & TGW-A & Toglorhoi \\
\hline TGW-A/5 & C1 & TGW-A & Toglorhoi \\
\hline TGW-A/3+4 & C1 & TGW-A & Toglorhoi \\
\hline TGS/surface & $\mathrm{C} 1$ & TGS & Toglorhoi (south) \\
\hline TAT-W/top & $\mathrm{C} 1$ & TAT & Tatatal Gol \\
\hline TAT-043 & $\mathrm{C} 1$ & TAT-E & Tatal Gol \\
\hline TAT-051/2 & C1 & TAT & Tatal Gol \\
\hline TAT-052/1 & $\mathrm{C} 1$ & TAT & Tatal Gol \\
\hline TAT-042 & $\mathrm{C} 1$ & TAT & Tatal Gol \\
\hline TAT-E/22 & C1 & TAT-E & Tatal Gol \\
\hline TAT-E/27 & $\mathrm{C} 1$ & TAT-E & Tatal Gol \\
\hline TAT-E/32 & C1-D & TAT-E & Tatal Gol \\
\hline RHN-A/7 & $\mathrm{C} 1$ & RHN-A & Huch Teeg \\
\hline RHN-023 & $\mathrm{C} 1$ & RHN & Huch Teeg \\
\hline HTSE-009+013 & $\mathrm{C} 1$ & HTSE & Hotuliin Teeg (southeast) \\
\hline HTSE-009/1 & $\mathrm{C} 1$ & HTSE & Hotuliin Teeg (southeast) \\
\hline HTSE-009/2 & $\mathrm{C} 1$ & HTSE & Hotuliin Teeg (southeast) \\
\hline HTSE-009/3 & $\mathrm{C} 1$ & HTSE & Hotuliin Teeg (southeast) \\
\hline HTSE-010 & $\mathrm{C} 1$ & HTSE & Hotuliin Teeg (southeast) \\
\hline HTSE-013/5 & $\mathrm{C} 1$ & HTSE & Hotuliin Teeg (southeast) \\
\hline HTE-057 & $\mathrm{C} 1$ & HTE & Hotuliin Teeg (east) \\
\hline HTS-011/1 (=HTS-056/1+2) & C1-D & HTS & Hotuliin Teeg (south) \\
\hline HTS-012 (=HTS-056/3) & C1-D & HTS & Hotuliin Teeg (south) \\
\hline HTE-009 & $\mathrm{D}$ & HTE & Hotuliin Teeg (east) \\
\hline HTE-008 & $\mathrm{D}$ & HTE & Hotuliin Teeg (east) \\
\hline HTE-014-018 & $\mathrm{D}$ & HTE & Hotuliin Teeg (east) \\
\hline $\mathrm{UNCH}-\mathrm{A} / 3+4$ & $\mathrm{D}$ & UNCH-A & Unkheltseg \\
\hline
\end{tabular}

ANOVA, post hoc tests were performed (Tukey's significant difference and Hochberg's GT2 when equal variances are assumed; and Games-Howell when not). All analyses were accomplished using exclusively localities with sample sizes larger than four specimens. The statistical analyses were done using SPSS version 15.0.1 (SPSS, 2006).

The specimens described and figured are integrated in the collections of the Natural History Museum in Vienna (Austria) abbreviated as NHMW.

\section{GEOLOGICAL SETTING}

The Valley of Lakes is part of the Pre-Altai depressions and is situated between the Gobi Altai Mountains in the south and the Khangai Mountains in the north (Figure 1). This wide depression of approximately $500 \mathrm{~km}$ in W-E direction is filled above a Proterozoic to Paleozoic basement with terrestrial sediments, ranging in age from the Cretaceous to the Quaternary. In the Taatsiin Gol and Taatsiin Tsagaan Nuur area, at an eastern longitude of $101^{\circ}$ to $102^{\circ}$ and a northern latitude of $45^{\circ}$ to $46^{\circ}$, the Cenozoic sediment sequence of the Hsanda Gol and Loh Fms is associated with basalts. ${ }^{40} \mathrm{Ar} / 39 \mathrm{Ar}$ datings of the basalt flows define 
TABLE 2. Descriptive statistics of the upper molars of Yindirtemys deflexus from the Valley of Lakes (Mongolia). Abbreviations: T.N., total number of teeth; N., number of teeth measured; min, minimum; max, maximum, $\delta$, standard deviation. For abbreviations of assemblages see Table 1.

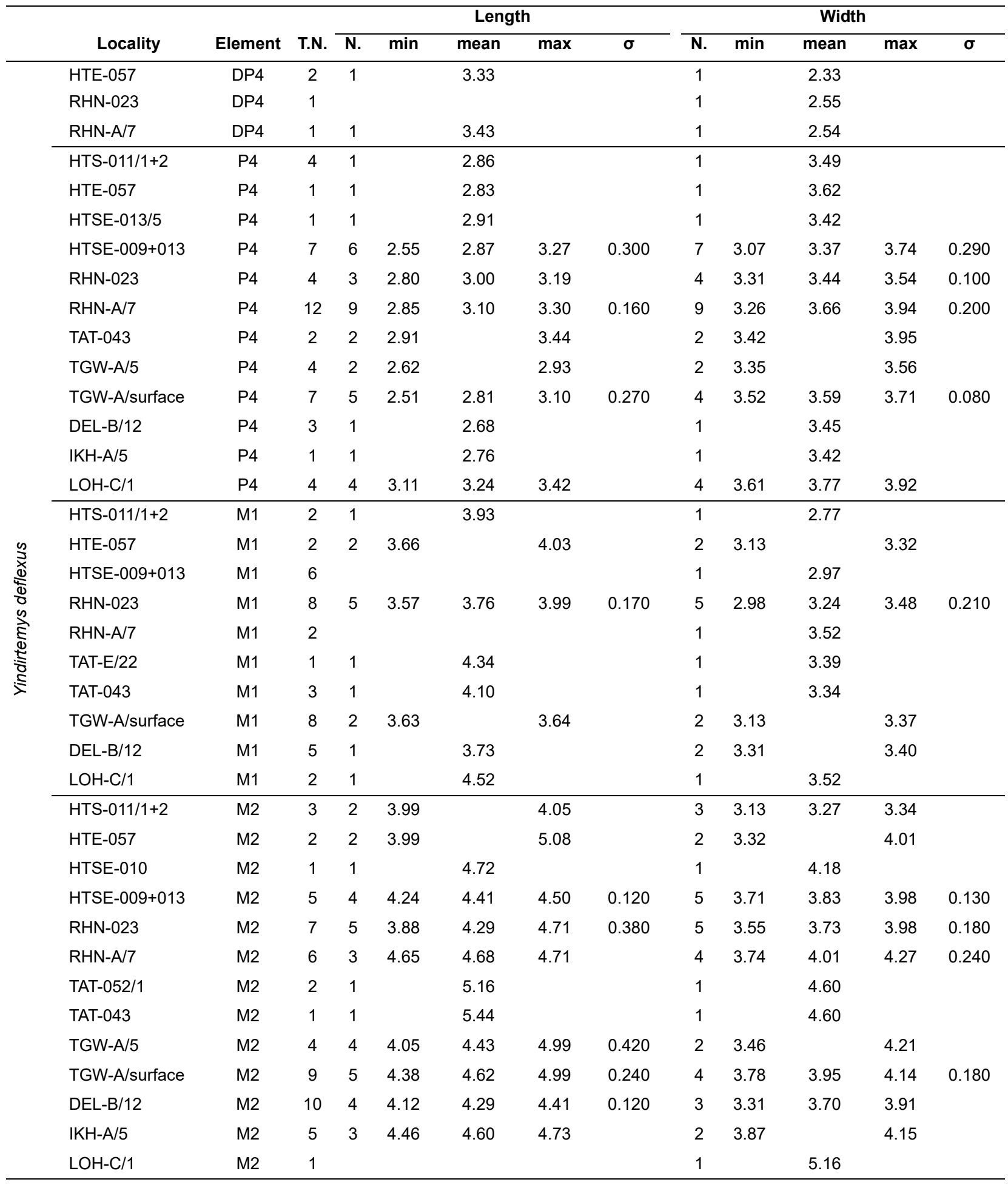


TABLE 2 (continued).

\begin{tabular}{|c|c|c|c|c|c|c|c|c|c|c|c|c|c|}
\hline & \multirow[b]{2}{*}{ Locality } & \multirow[b]{2}{*}{ Element } & \multirow[b]{2}{*}{ T.N. } & \multicolumn{5}{|c|}{ Length } & \multicolumn{5}{|c|}{ Width } \\
\hline & & & & $\mathbf{N}$. & $\min$ & mean & $\max$ & $\sigma$ & $\mathbf{N}$. & $\min$ & mean & $\max$ & $\sigma$ \\
\hline \multirow{14}{*}{ 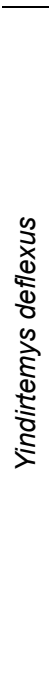 } & HTS-011/1+2 & M3 & 2 & 1 & & 5.04 & & & 1 & & 4.89 & & \\
\hline & HTE-057 & M3 & 2 & 1 & & 5.15 & & & 1 & & 4.50 & & \\
\hline & HTSE-009/1 & M3 & 2 & 1 & & 4.20 & & & & & & & \\
\hline & HTSE-009+013 & M3 & 9 & 9 & 4.51 & 4.91 & 5.84 & 0.430 & 8 & 4.18 & 4.43 & 4.83 & 0.200 \\
\hline & $\mathrm{RHN}-023$ & M3 & 2 & 2 & 4.80 & & 5.26 & & 2 & 3.94 & & 4.09 & \\
\hline & RHN-A/7 & M3 & 6 & 6 & 4.79 & 5.07 & 5.38 & 0.220 & 6 & 4.06 & 4.42 & 4.74 & 0.230 \\
\hline & TAT-E/27 & M3 & 1 & 1 & & 5.44 & & & 1 & & 4.36 & & \\
\hline & TAT-052/1 & M3 & 2 & 2 & 4.75 & & 5.81 & & 2 & 4.52 & & 4.85 & \\
\hline & TAT-043 & M3 & 3 & 3 & 5.19 & 5.45 & 5.70 & & 3 & 4.45 & 4.69 & 4.82 & \\
\hline & TGW-A/5 & M3 & 2 & 2 & 4.69 & & 4.87 & & 2 & 3.94 & & 4.03 & \\
\hline & TGW-A/surface & M3 & 6 & 4 & 4.48 & 4.58 & 4.68 & 0.090 & 4 & 4.01 & 4.06 & 4.11 & 0.040 \\
\hline & DEL-B/12 & M3 & 7 & 5 & 4.24 & 4.65 & 5.02 & 0.290 & 4 & 3.93 & 4.13 & 4.34 & 0.190 \\
\hline & $\mathrm{IKH}-\mathrm{A} / 5$ & M3 & 2 & 2 & 4.69 & & 4.95 & & 2 & 3.92 & & 4.40 & \\
\hline & $\mathrm{LOH}-\mathrm{C} / 1$ & M3 & 4 & 3 & 5.11 & 5.26 & 5.57 & & 3 & 4.30 & 4.54 & 4.68 & \\
\hline
\end{tabular}

at least two groups of Oligocene basalts (basalt I: 31.5 m.y.; basalt II: 27-28 m.y.) and a middle Miocene basalt (basalt III: $\sim 13$ m.y.) (Daxner-Höck et al., 1997; Höck et al., 1999). In this area the exposed sediment sequence of the Hsanda Gol and Loh formations is locally very rich in Oligocene and Miocene fossils. It forms the biological basis for the biozonation (Daxner-Höck et al., 1997; Höck et al., 1999) and updates the Mongolian biozones (Daxner-Höck and Badamgarav, 2007; Daxner-Höck et al., 2010, 2014, 2015).

Our study focuses on the late Oligocene (biozones $C$ and $C 1$ ), the Oligocene-Miocene transition (biozone C1-D), and the earliest Miocene (biozone D). In the sections Abzag Ovo (ABO-A) and Unzing Khurem (TAR-A), fossils which characterize biozone $C$ were recovered below basalt II (132/97 ABO: $27.0 \pm 0.9$ M.y.) and above basalt II (M56/96 TAR: $27.4 \pm 0.4$ M.y.). Accordingly, the age of the assemblages of biozone $\mathrm{C}$ is late Oligocene (Daxner-Höck et al., 2010, 2014, 2015). From several sections (Tatal Gol, Toglorhoi, Ikh Argalatyn Nuru, Huch Teeg) biozone $\mathrm{C} 1$ faunas are known immediately above biozone $C$, and biozone $C 1-D$ above biozone C1. In Hotuliin Teeg, biozones C1 and C1$\mathrm{D}$ are topped by biozone $\mathrm{D}$. The Mongolian biozones C1 and C1-D correlate biostratigraphically with the Chinese Mammal Age Tabenbulukian (late late Oligocene), and biozone $D$ with the Xieijan (early Miocene) (Figure 3).

\section{SYSTEMATIC PALAEONTOLOGY}

Order RODENTIA Bowdich, 1821 Family CTENODACTYLIDAE Zittel, 1893 Subfamily TATAROMYINAE Lavocat, 1961

Genus YINDIRTEMYS Bohlin, 1946

Type Species. Yindirtemys grangeri Bohlin, 1946

Yindirtemys deflexus (Teilhard de Chardin, 1926)

Figures 4.1-18, 5.1-21

1926

1942

Tataromys deflexus Teilhard de Chardin, $p$. 28, p.31, fig. 15B; pl. IV, fig. 3.

1946

Tataromys deflexus Teilhard de Chardin and Leroy, p. 25, 89.

1951 Tataromys deflexus Stehlin and Schaub, p. 125, fig. 181.

1951 Tataromys sp. Stehlin and Schaub, p. 289, fig. 496.

1958 Tataromys sp. Schaub, p. 781, fig. 211.

1968 Tataromys deflexus Mellett, p. 6, 10.

1974 Tataromys deflexus Kowalski, p. 160-161, pl. XLVII, fig. 1.

1974 Tataromys gobiensis Kowalski, p. 162.

1981 Tataromys deflexus Wang, Chang, Meng, and Chen, p. 29.

1993 Yindirtemys sajakensis Bendukidze, p. 6063, pl. 20, figs 2, 3, 4, 5, 6, 7; pl. 21.

1994 Yindirtemys deflexus Wang, p. 37, figs 2a, b.

1997 Yindirtemys deflexus Wang, p. 30-34.

1997 Yindirtemys gobiensis Wang, p. 34-35.

1997 Yindirtemys sajakensis Bendukidze, p. 207. 
TABLE 3. Descriptive statistics of the lower molars of Yindirtemys deflexus from the Valley of Lakes (Mongolia). Abbreviations: T.N., total number of teeth; N., number of teeth measured; min, minimum; max, maximum, $\delta$, standard deviation. For abbreviations of assemblages see Table 1.

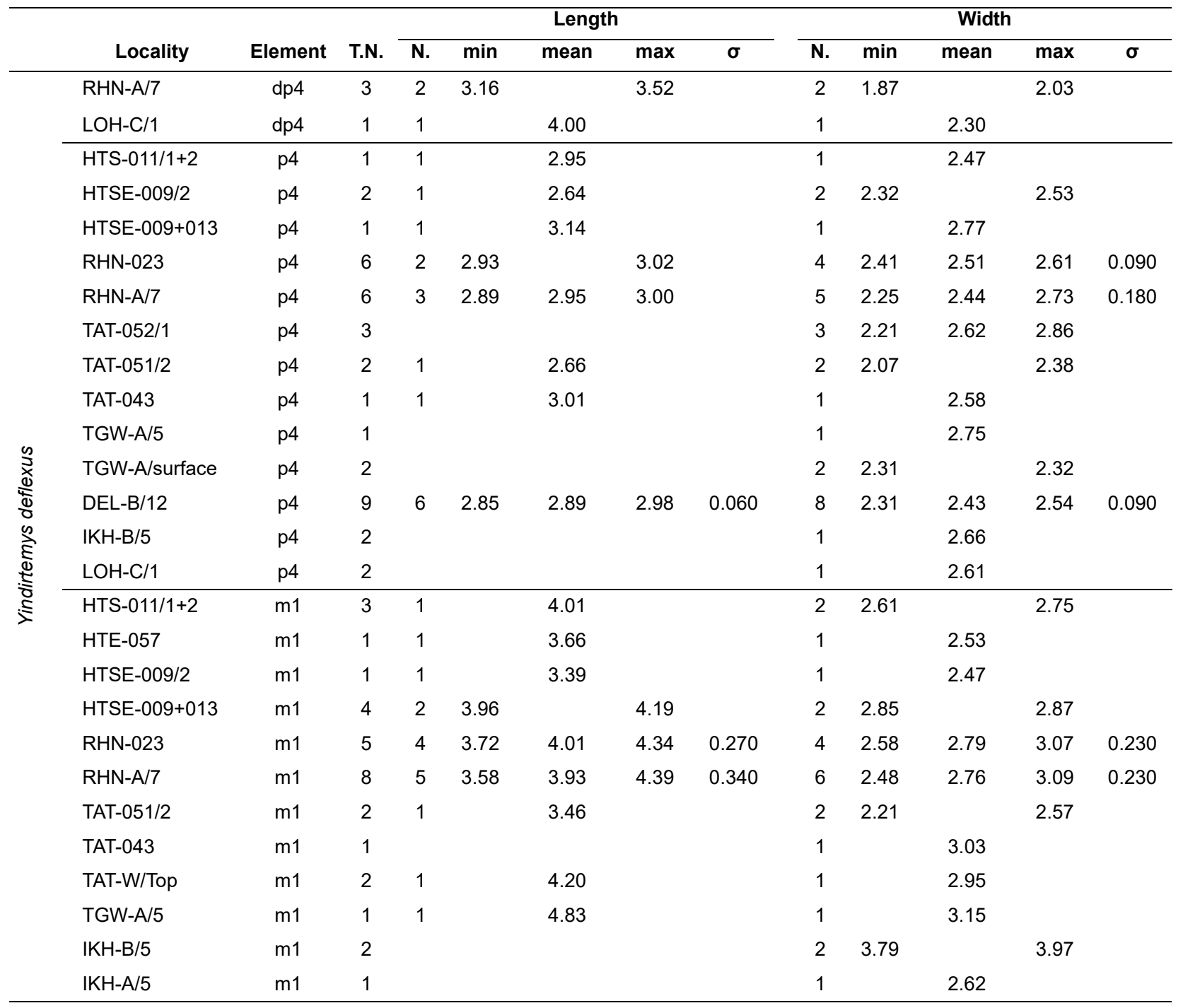

1999 Yindirtemys deflexus Höck, Daxner-Höck, Schmid, Badamgarav, Frank, Furtmüller, Montag, Barsbold, Khand, and Sodov, p. 116-118.

$2004 \quad$ Yindirtemys sajakensis Lopatin, p. 298.

2006 Yindirtemys deflexus Vianey-Liaud, SchmidtKittler, and Marivaux, p. 164-165, 190-191.

2007 Yindirtemys deflexus Schmidt-Kittler, Vianey-Liaud and Marivaux, p. 191-201.

2007 Yindirtemys deflexus Daxner-Höck and Badamgarav, p. 15-16, 18.

2009 Yindirtemys deflexus Bendukidze, de Bruijn, and Van den Hoek Ostende, p. 351-352, 356, 368-369.
2010 Yindirtemys deflexus Daxner-Höck, Badamgarav, and Erbajeva p. 358, 362-363, fig. 6.1-6.2.

2014 Yindirtemys deflexus Gomes Rodrigues, Marivaux, and Vianey-Liaud p. 7.

Holotype. Fragment of a right maxilla with M2 and M3. Teilhard de Chardin (1926: figure 15B).

Type Locality. Saint Jacques (Inner Mongolia, China).

Material and Measurements. Tables 2 and 3. Stratigraphic Range in Mongolia. Upper part of the late Oligocene, Mongolian biozone C1; Hsanda Gol and Loh formations.

Geographic Distribution. Valley of Lakes (Mongolia), North Aral region (Kazakhstan), Gansu 
TABLE 3 (continued).

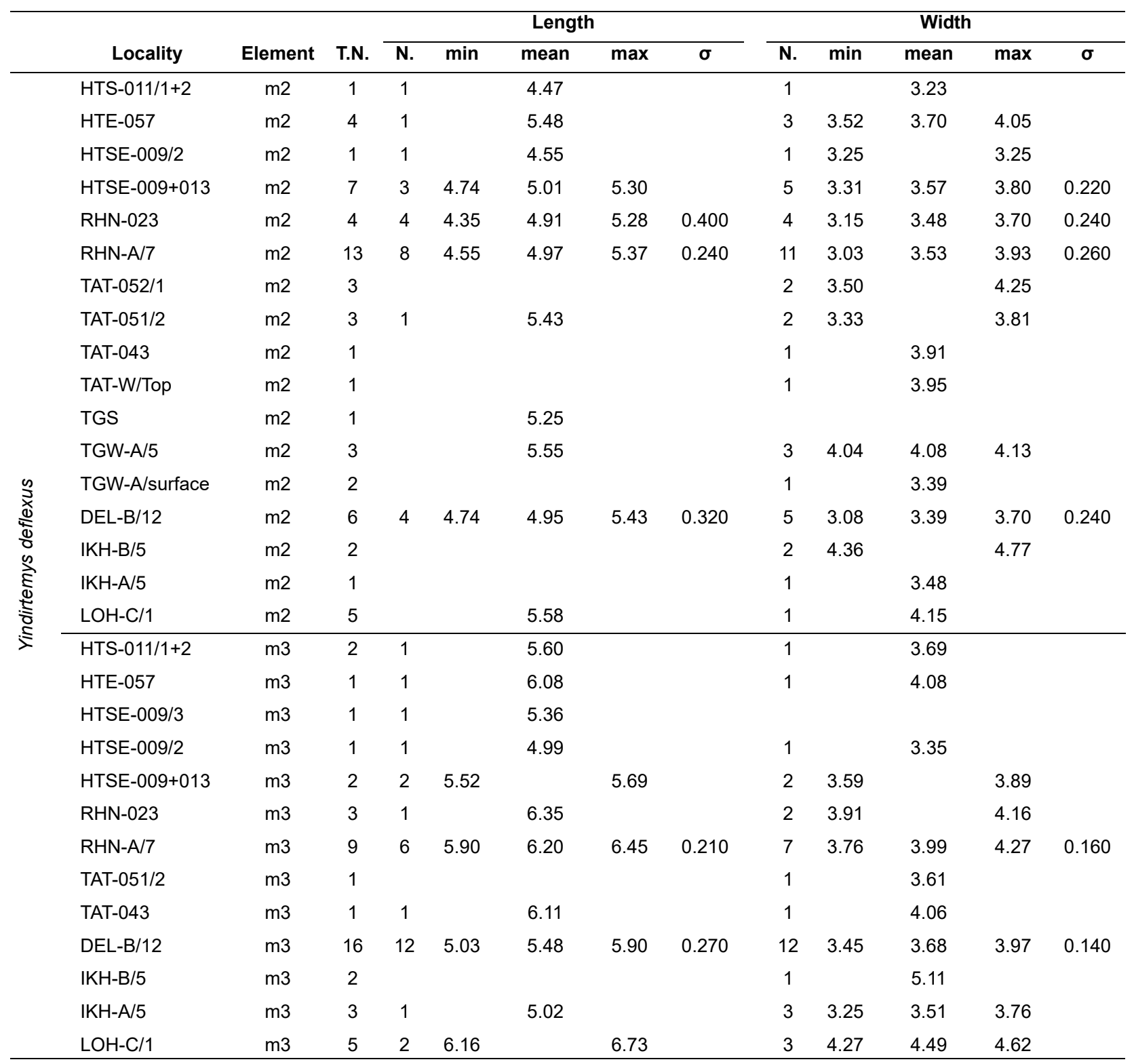

province (China), Ulantatal area and Saint Jacques (Inner Mongolia, China).

Description. The description of Yindirtemys deflexus is based on the very rich material from the locality Huch Teeg (RHN-A/7) in Mongolia.

P4. The labial anteroloph is incipient (one out of 10 specimens), short (seven out of 10) or medium (two out of 10). The lingual anteroloph is short in one, incipient in three, and absent in six specimens. Out of 10 specimens, the labial posteroloph is short in six, medium in three, and long and connected to the paracone in one. The labial crest of the metaloph is long and connected to the posteroloph (four out of 10), or there is a double crest (six out of 10). The lingual posteroloph is incipient in one specimen, short in one, and long and connected to the protocone in seven specimens. The lingual crest of the metaloph is short (one out of nine specimens), or it is long and connected to the posteroloph (eight out of nine) (Figures 4.9-11).

M1. There are only two teeth, M1s, of which one is heavily worn. In the less worn specimen the labial anteroloph is of medium length. The lingual anteroloph is absent. There is an incipient forward paracone spur. The protoloph is anterior and transversely directed to the protocone.

M2. The labial anteroloph is short (one out of four), medium (one out of four), or it is connected to the 
TABLE 4. Descriptive statistics of the upper and lower molars of Yindirtemys suni from the Valley of Lakes (Mongolia) and the holotype from the Xiejia Formation of Xiejia, Quinghai (IVPP V 5992). Abbreviations: T.N., total number of teeth; N., number of teeth measured; min, minimum; max, maximum, $\delta$, standard deviation. For abbreviations of assemblages see Table 1.

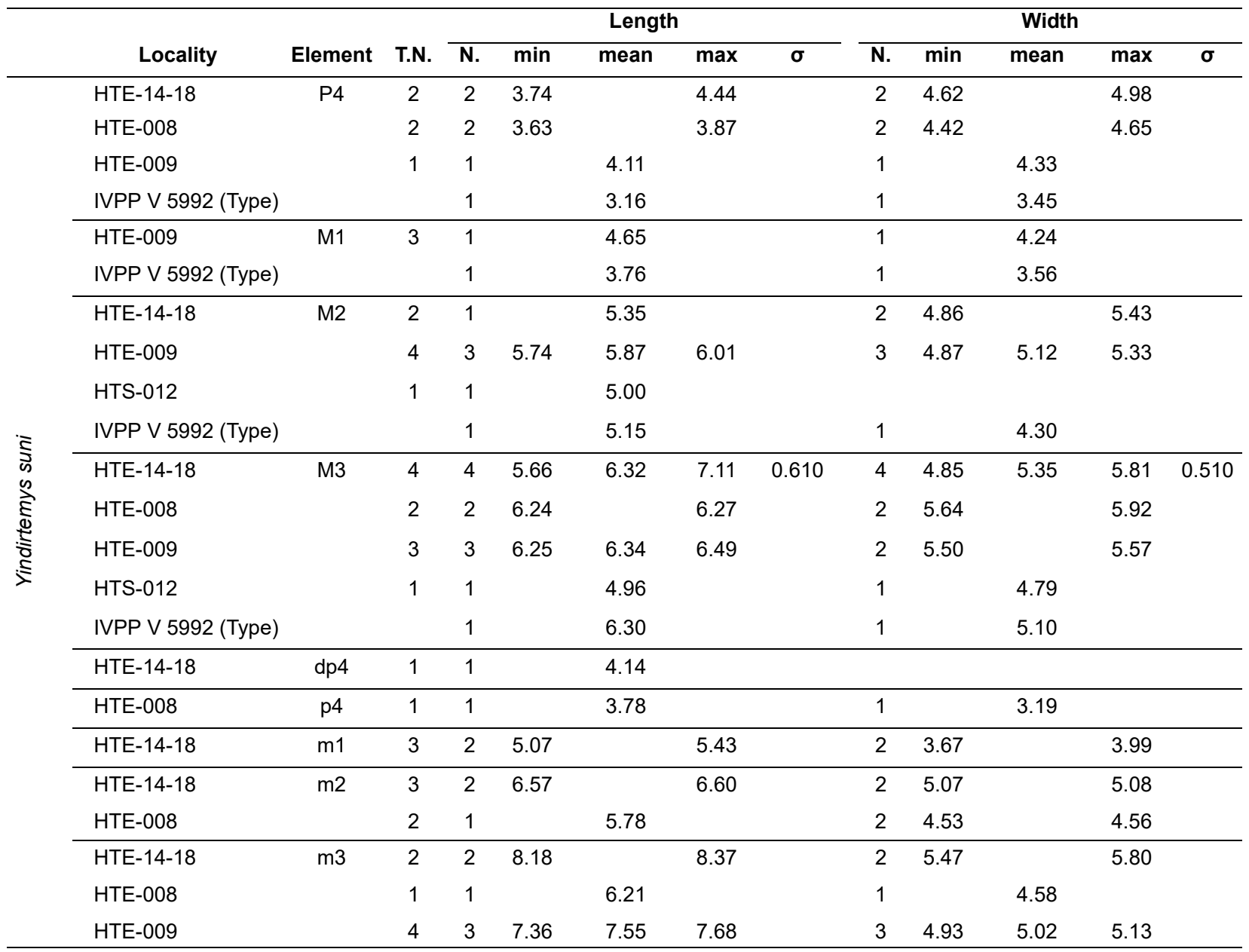

paracone (two out of four). The lingual anteroloph is absent (four out of four). A forward paracone spur is connected to the labial anteroloph in three (out of five) specimens. The protoloph is anterior (four out of four) and runs proverse in two specimens and runs transverse in the remaining two. In one out of four, there is a short and wide crochet, in the remaining three the anticrochet is connected to the posteroloph. The metaloph is transverse (two out of four) or points backwards (two out of four), and it is transversely directed in one and proverse in three specimens (Figure 4.13).

M3. The labial anteroloph is connected to the paracone in three specimens and is of medium length in two teeth. The lingual anteroloph is incipient in two and absent in three specimens. The anterior groove is always retroverse, and it is deep (three out of five specimens) or shallow (two out of five). A forward paracone spur is connected to the labial anteroloph in one out of five specimens. The protoloph is anterior in four specimens and double in one. It is transversely directed in three and proverse in the remaining two. In one out of five, there is an incipient crochet, in two the crochet is well developed, and in the remaining two there is a deflexus structure and anticrochet. The sinus is retroverse (two out of six specimens), transverse (two out of six) or proverse, (two out of six) and always deep. The metaloph is transverse (three out of five) or points backwards (two out of five), and it is transversely directed in one specimen and proverse in four. The morphology of the teeth is swollen and bulky (three out of five) or the teeth are straighter, like M2 (two out of five) (Figure 4.5-7, 4.12).

DP4. There is only one specimen. The labial anteroloph is short. The lingual anteroloph is absent. There is an incipient anterior paracone spur. The 
TABLE 5. Descriptive statistics of the upper and lower molars of Yindirtemys birgeri from the Aral Formation (Kazakhstan) and the locality Tatal Gol (TAT-051/2; 2012/0060/0001) in the Valley of Lakes (Mongolia). Abbreviations: T.N., total number of teeth; N., number of teeth measured; min, minimum; max, maximum, $\delta$, standard deviation. The data on Y. birgeri from the Aral Formation are from Bendukidze et al. (2009).

\begin{tabular}{|c|c|c|c|c|c|c|c|c|c|c|c|c|}
\hline & \multirow[b]{3}{*}{ Country } & \multirow[b]{3}{*}{ Element } & \multirow{2}{*}{\multicolumn{5}{|c|}{ Length }} & \multirow{2}{*}{\multicolumn{5}{|c|}{ Width }} \\
\hline & & & & & & & & & & & & \\
\hline & & & N. & $\min$ & mean & $\max$ & $\sigma$ & N. & $\min$ & mean & $\max$ & $\sigma$ \\
\hline \multirow{9}{*}{$\begin{array}{l}\text { Yindirteys } \\
\text { birgeri }\end{array}$} & \multirow{8}{*}{$\begin{array}{l}\text { Aral Formation } \\
\text { (Kazakhstan) }\end{array}$} & $\mathrm{P} 4$ & 6 & 2.3 & 2.47 & 2.5 & 0.08 & 6 & 2.75 & 2.9 & 3 & 0.12 \\
\hline & & M1 & 3 & 2.87 & 2.96 & 3 & 0.08 & 3 & 2.37 & 2.42 & 2.5 & 0.07 \\
\hline & & M2 & 6 & 3.5 & 3.59 & 3.7 & 0.08 & 6 & 2.87 & 3.14 & 3.37 & 0.19 \\
\hline & & M3 & 7 & 3.62 & 3.91 & 4.12 & 0.19 & 7 & 2.5 & 3.23 & 3.5 & 0.38 \\
\hline & & $\mathrm{p} 4$ & 6 & 2.2 & 2.41 & 2.75 & 0.19 & 6 & 1.85 & 2.03 & 2.2 & 0.16 \\
\hline & & $\mathrm{m} 1$ & 2 & 3.1 & 3.18 & 3.25 & 0.11 & 2 & 2 & 2.2 & 2.4 & 0.28 \\
\hline & & $\mathrm{m} 2$ & 3 & 3.95 & 4.28 & 4.5 & 0.29 & 3 & 2.6 & 2.8 & 2.9 & 0.17 \\
\hline & & m3 & 5 & 4.8 & 4.88 & 5 & 0.08 & 5 & 3 & 3.09 & 3.25 & 0.1 \\
\hline & & $\mathrm{P} 4$ & 2 & 2.53 & & 2.54 & & 2 & 2.99 & & 3.02 & \\
\hline TAT-051/2 & Valley of Lakes & M2 & 1 & & 3.31 & & & 2 & 3.25 & & 3.26 & \\
\hline $2012 / 0060 / 0001$ & (Mongolia) & M3 & 2 & 3.35 & & 3.75 & & 2 & 3.48 & & 3.52 & \\
\hline
\end{tabular}

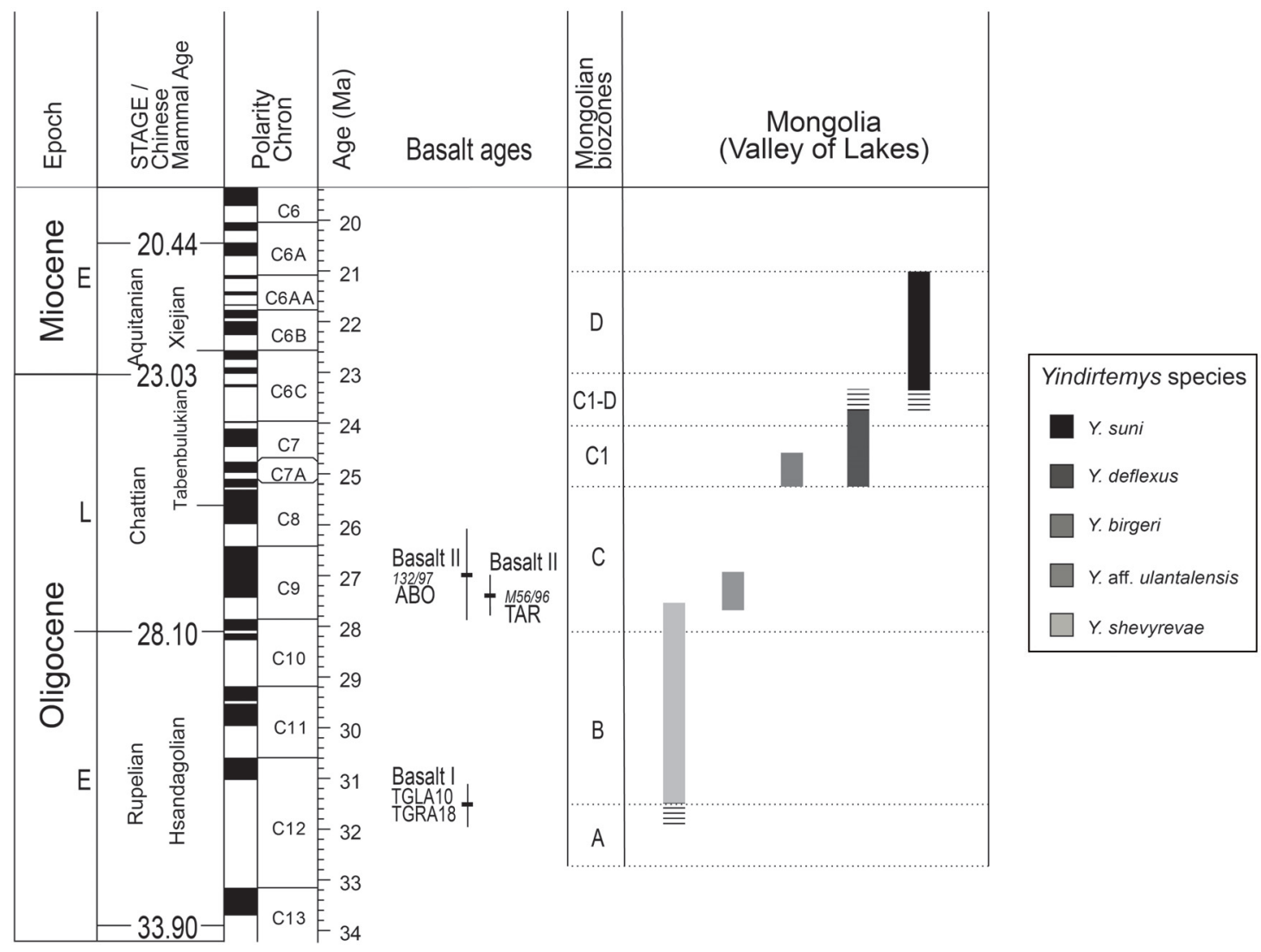

FIGURE 3. Stratigraphic ranges of the Yindirtemys species from the Valley of Lakes (Mongolia). Stratigraphic chart (modified from Daxner-Höck et al., 2015) including the geological time scale (Gradstein et al., 2004), basalt ages and Mongolian biozones A - D (Höck et al., 1999), the lower boundary of the Xiejian Chinese Mammal Age after Meng et al. (2006). 


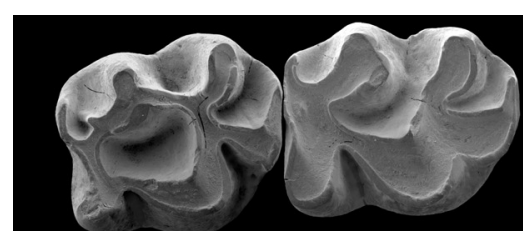

15.
$1 \mathrm{~mm}$

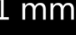

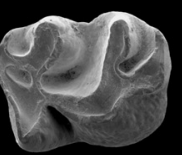

16.

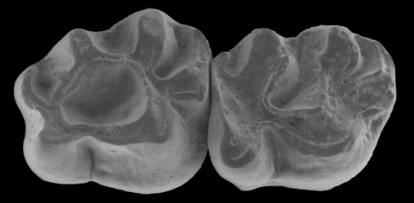

17.

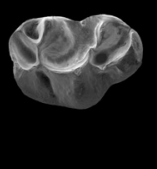

18.
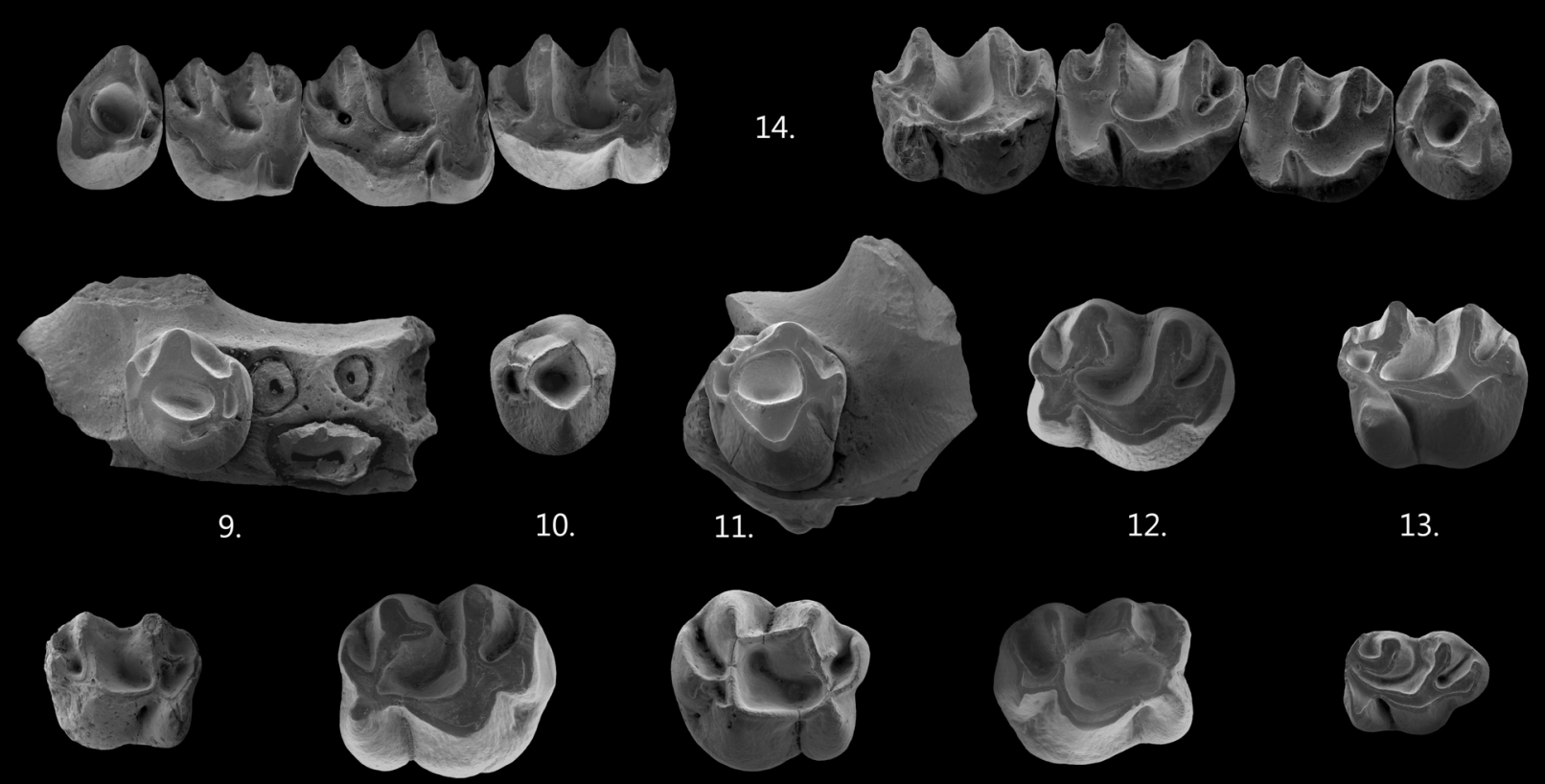

5.

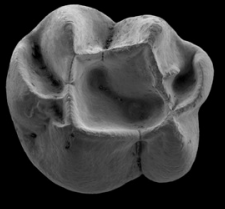

6.

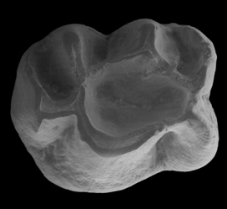

7.

8.
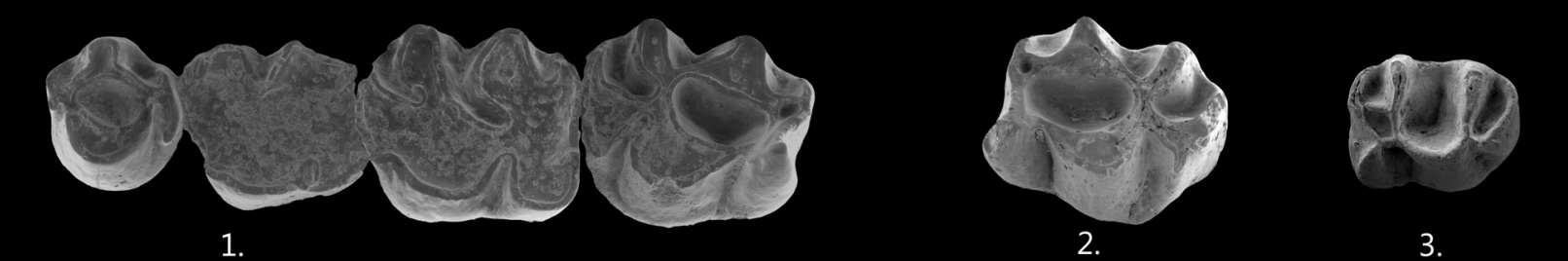

FIGURE 4. Upper molars of Yindirtemys deflexus from the Valley of Lakes (Mongolia). Nos. 1-18. From Loh: 1) LOH-C/ 1-NHMW 2006./0083/0002 P4-M3 left; 2) LOH-C/1-NHMW 2006/0083/0007 M3 right. From Del: 3) DEL-B/12-NHMW 2006/0089/0006 M2 right; 4) DEL-B/12-NHMW 2006/0089/0033 M1 left. From Huch Teeg: 5) RHN-A/7-NHMW 2006/ 0090/0009 M3 right; 6) RHN-A/7-NHMW 2006/0090/0010 M3 left; 7) RHN-A/7-NHMW 2006/0090/0012 M3 left; 8) RHN-A/7-NHMW 2006/0090/0015 DP4 right; 9) RHN-A/7-NHMW 2006/0090/0039 P4 left; 10) RHN-A/7-NHMW 2006/ 0090/0045 P4 right; 11) RHN-A/7-NHMW 2006/0090/0047 P4 right; 12) RHN-A/7-NHMW 2006/0090/0052 M3 right; 13) RHN-A/7-NHMW 2006/0090/0053 M2 right. From Toglorhoi: 14) TGW-A/surface -NHMW 2006/0086/0005 Complete maxilla. From Tatal Gol: 15) TAT-043-NHMW 2012/0034/0005 M2-M3 right. From Huch Teeg: 16) RHN-023NHMW 2012/0038/0014 M1 right. From Hotuliin Teeg: 17) HTSE-009+013-NHMW 2012/0039/0015 M2-M3 right; 18) HTE-057-NHMW 2012/0045/0002 DP4 left. All teeth same magnification. Scale bar equals $1 \mathrm{~mm}$.

protoloph is anteriorly extended from the protocone and is connected to the anterocone. An anticrochet is connected to the posteroloph. The metaloph points backwards (Figure 4.8).

p4. The anterior sinusoid is always triangular and in three out of five bears a strong central furrow. The hypoconid is present in two and absent in three specimens. The hypolophid is present (three out of five specimens), double (one out of five), or absent (one out of five). In one out of four there is a hypoconulid (Figure 5.10, 5.12-13).

$\mathbf{m 1}$. The anterior cingulid is short in three specimens and long in four. In all specimens the metalophid $\mathrm{I}$ is well connected to the protoconid. The mesolophid is connected to the metaconid (six out of six specimens). The sinusid is M-shaped. The hypoconulid is always present (seven out of seven). The hypolophid is connected to the anterior arm of the hypoconid (four out of five) or is 


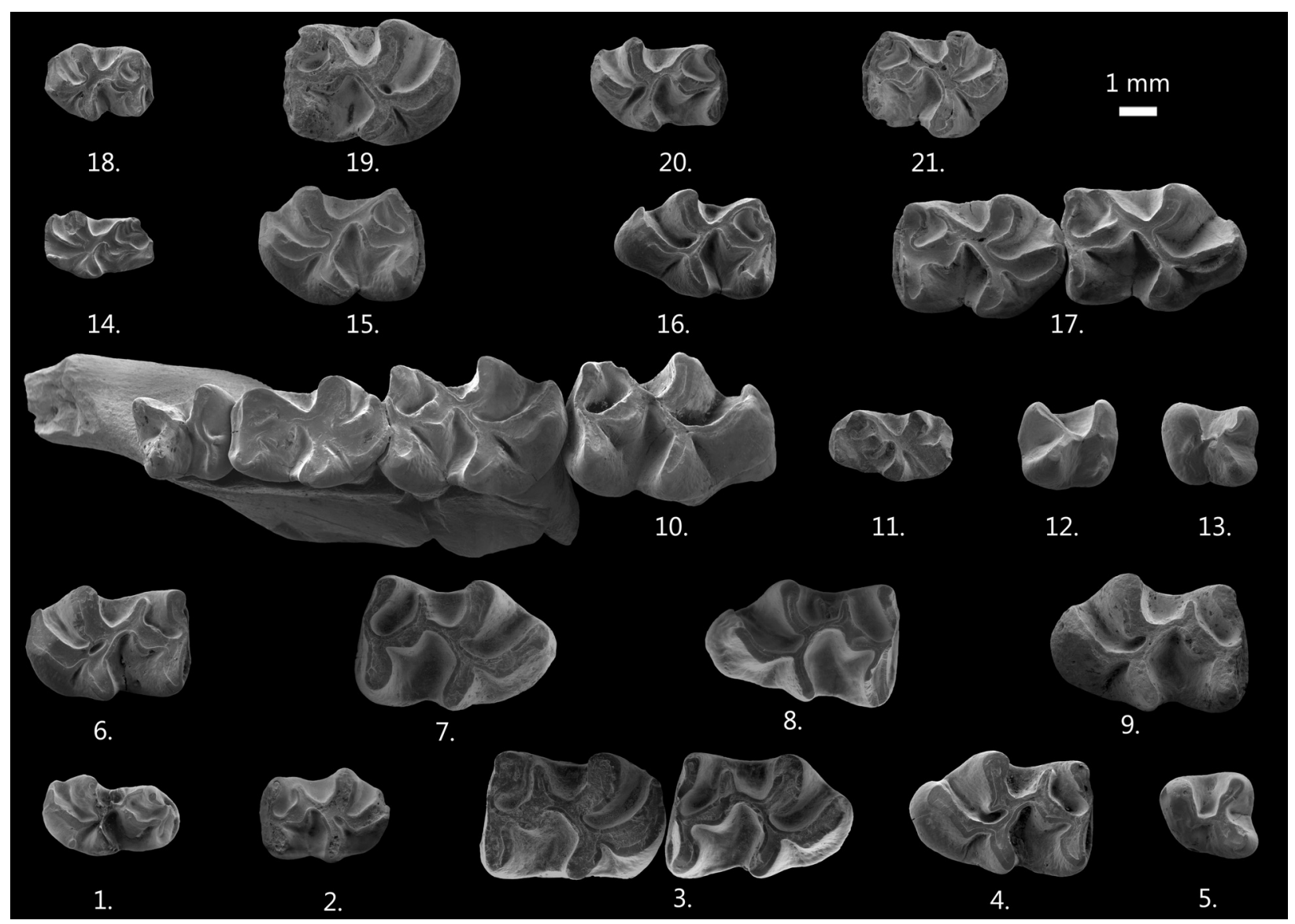

FIGURE 5. Lower molars of Yindirtemys deflexus from the Valley of Lakes (Mongolia). Nos. 1-21. From Loh: 1) LOHC/1-NHMW 2006/0083/0015 dp4 right. From Ikh Argalatyn Nuru: 2) IKH-A/5-NHMW 2009/0148/0011 m1 left. From Del: 3) DEL-B/12-NHMW 2006/0089/0001 m2-m3 left; 4) DEL-B/12-NHMW 2006/0089/0002 m3 right; 5) DEL-B/12NHMW 2006/0089/0018 p4 right; 6) DEL-B/12-NHMW 2006./0089/0025 m2 left; 7) DEL-B/12-NHMW 2006/0089/ 00027 m3 left; 8) DEL-B/12-NHMW 2006/0089/0029 m3 left; 9) DEL-B/12-NHMW 2006/0089/0041 m3 right. From Huch Teeg: 10) RHN-A/7-NHMW 2006/0090/0001 p4-m3 left; 11) RHN-A/7-NHMW 2006/0090/0004 dp4 left; 12 ) RHN-A/7-NHMW 2006/0090/0005 p4 left; 13) RHN-A/7-NHMW 2006/0090/0019 p4 right; 14) RHN-A/7-NHMW 2006/ 0090/0021 dp4 right; 15) RHN-A/7- NHMW 2006/0090/0031 m2 right; 16) RHN-A/7- NHMW 2006/0090/0032 m3 right; 17) RHN-A/7-NHMW 2006/0090/0037 m2-m3 left. From Tatal Gol: 18) TAT-051/2-NHMW 2012/0035/0005 m1 right. From Huch Teeg: 19) RHN-023-NHMW 2012/0038/0023 m2 left; 20) RHN-023-NHMW 2012/0038/0027 m1 right. From Hotuliin Teeg: 21) HTSE-009+013-NHMW 2012/0039/0020 m1 left. All teeth same magnification. Scale bar: 1 $\mathrm{mm}$.

directly connected to the hypconid (one out of five) (Figure 5.10).

m2. The anterior cingulid is short (two out of 11 specimens) or long (nine out of 11). The metalophid $\mathrm{I}$ is well connected to the protoconid in all the specimens. The mesolophid is connected to the metaconid in 10 specimens, is medium long in one and short in one. The sinusid is M-shaped. In 11 out of 12 specimens the hypoconulid is present; the remaining specimen bears two hypoconulids. The hypolophid is always connected to the anterior arm of the hypoconid (Figure 5.10, 5.15, 5.17).

$\mathrm{m3}$. The anterior cingulid is short in two specimens and long in four. The metalophid I is well connected to the protoconid. The mesolophid is long and connected to the metaconid (seven out of eight specimens) or is short (one out of eight). The sinusid is M-shaped. The hypoconulid is always present. In all specimens the hypolophid is connected to the anterior arm of the hypoconid (Figure 5.10, 5.1617).

dp4. The anterior cingulid is short (one out of two specimens) or long (one out of two). The metalophid $\mathrm{I}$ is connected to the protoconid. The trigonid basin is wide, shallow, and it is close. The mesolophid is long and connected to the metaconid. The sinusid is M-shaped in one specimen and crescent-shaped in the other. In one specimen there is 


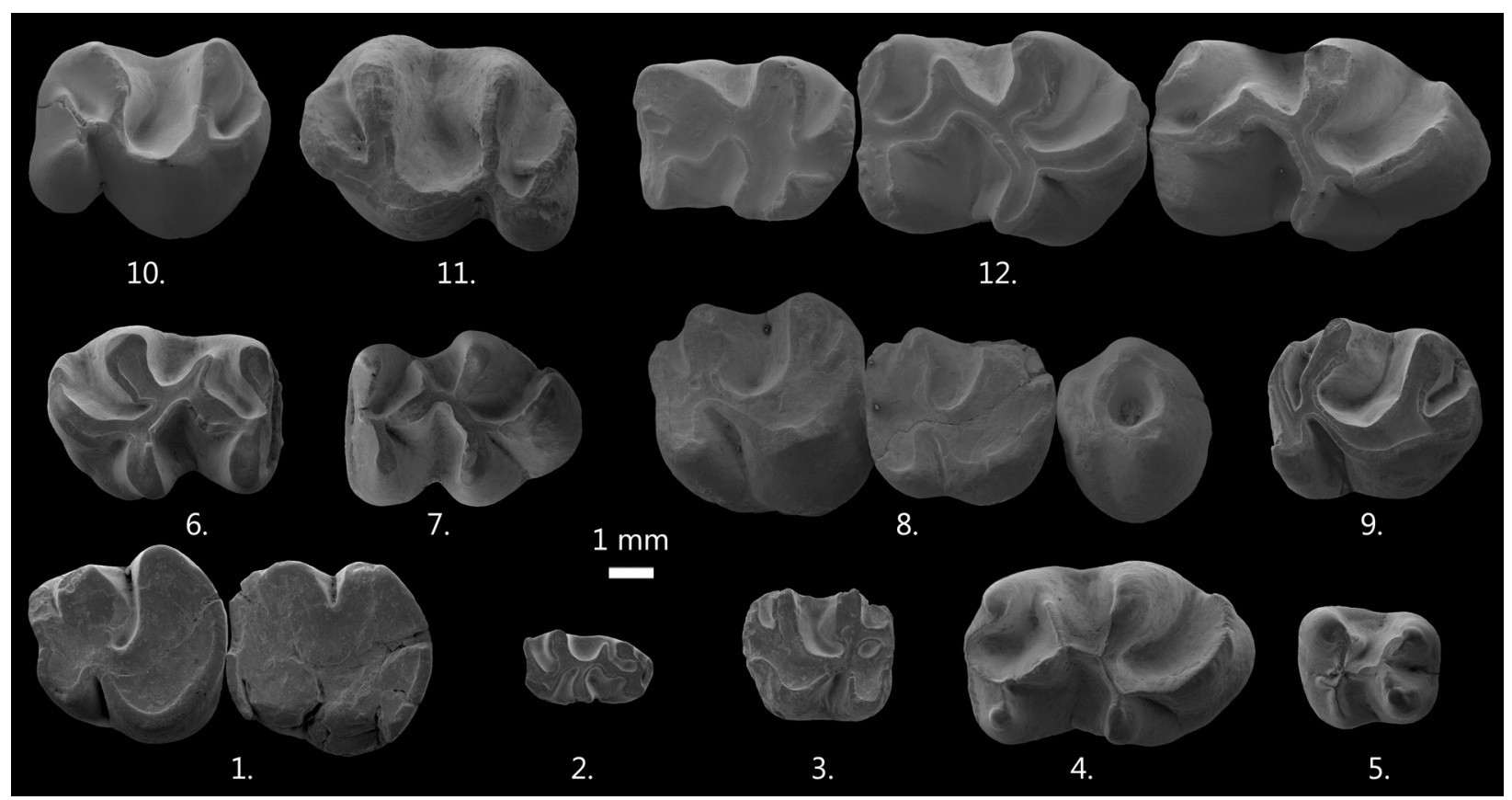

FIGURE 6. Yindirtemys suni from the Valley of Lakes (Mongolia). Nos. 1-12. From Hotuliin Teeg (south): 1) HTS-056/ 3-NHMW 2012/0047/0001 M2-M3 right. From Unkheltseg: 2) UNCH-A/4-NHMW 2006/0088/0001 dp4 right; 3) UNCHA/4-NHMW 2006/0088/0003 M1 left. From Hotuliin Teeg (east): 4) HTE-009-NHMW2012/0033/0009 m3 left; 5) HTE008-NHMW 2012/0032/0004 p4 right; 6) HTE-008-NHMW 2012/0032/0005 m2 right; 7) HTE-008-NHMW 2012/0032/ 0006 m3 left; 8) HTE-014-018-NHMW 2012/0031/0001 P4-M2 right; 9) HTE-014-018-NHMW 2012/0031/0002 M2 right; 10) HTE-014-018-NHMW 2012/0031/0003 M3 right; 11) HTE-014-018-NHMW 2012/0031/0006 M3 left; 12) HTE-014-018-NHMW 2012/0031/0009 m1-m3 left. All teeth same magnification. Scale bar equals $1 \mathrm{~mm}$.

an incipient stylid in the sinusid. The hypoconulid is always present. In one out of two specimens there is a stylid in the hypoconulid (Figure 5.11, 5.14).

Remarks. The Yindirtemys material from the Valley of Lakes does not differ from the holotype of Yindirtemys deflexus from Saint Jacques (Teilhard de Chardin, 1926) and also resembles the specimens of $Y$. deflexus from the Ulantatal area (Vianey-Liaud et al., 2006).

Yindirtemys suni (Li and Qiu, 1980).

Figure 6.1-6.12

1980 Tataromyssuni Li and Qiu, p. 205-206, 212, fig. 7; pl. I, fig. 3.

1981 Tataromyssuni Wang, Chang, Meng, and Chen, p. 27, 29, 34.

1988 Tataromyssuni Qiu and Gu, p. 204-206, 211, pl. II, figs 1, 2, 3, 4, 10.

$1994 \quad$ Yindirtemys suni Wang, p. 37.

1997 Yindirtemys suni Wang, p. 35-37.

2007 Yindirtemys deflexus (pro-parte) SchmidtKittler, Vianey-Liaud, and Marivaux, p. 191201.

2007 Yindirtemys suni Daxner-Höck and Badamgarav, p. 16, 18.
Holotype. Right maxilla with P4-M3. Li and Qiu, (1980: fig. 7; pl. I, fig. 3).

Type Locality. Xiejia (Xining Basin, Qinghai, China).

Material and Measurements. Table 4.

Stratigraphic Range in Mongolia. The Oligocene-Miocene transition (biozone C1-D) and early Miocene (biozone D); Hsanda Gol and Loh Fms.

Geographic Distribution. Valley of Lakes (Mongolia), Inner Mongolia (China), Qinghai and Gansu province (China).

Description. The description is based on the material of Yindirtemys suni from the locality Hotuliin Teeg (HTE-014-018) in Mongolia.

P4. The labial anteroloph is medium long in one of two specimens or absent in the other. The lingual anteroloph is absent. The labial posteroloph is medium long. The lingual posteroloph is short. The lingual crest of the metaloph is long and connected to the posteroloph (Figure 6.8).

M2. The labial anteroloph is medium long (one out of two specimens) or long and connected to the paracone (one out of two). The lingual anteroloph is absent. The protoloph is anterior to the protocone. In one specimen the protoloph is transversely connected, and in the other one it is 


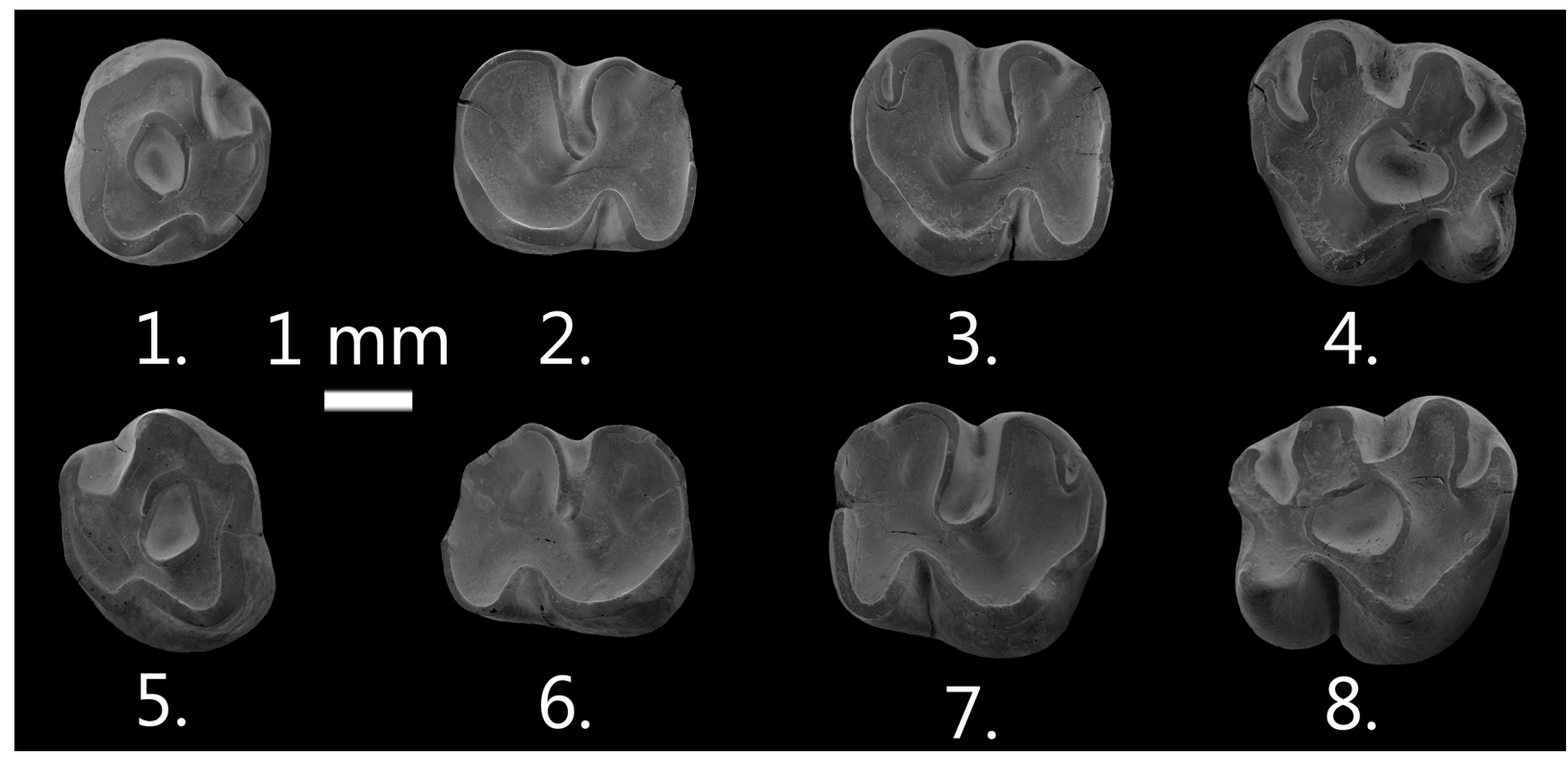

FIGURE 7. Complete maxilla of Yindirtemys birgeri from the locality of Tatal Gol (TAT-051/2- NHMW 2012/0060/0001) in the Valley of Lakes (Mongolia). Nos. 1-8. 1) P4 left; 2) M1 left; 3) M2 left; 4) M3 left; 5) P4 right; 6) M1 right; 7 ) M2 right; 8) M3 right. All teeth same magnification. Scale bar equals $1 \mathrm{~mm}$.

proverse. In one out of two, there is an incipient crochet; in the remaining one the anticrochet is connected to the posteroloph. In all the specimens the metaloph is anterior with respect to the hypocone and is connected transversely to it (Figure 6.8-9).

M3. The labial anteroloph is long and connected to the paracone (one out of four specimens) or it is medium long (three out of four). The lingual anteroloph is absent. The anterior groove is absent in three and shallow in the remaining specimen. The protoloph is always anterior to the protocone, and it runs transversely (three out of four) or proverse (one out of four). The sinus is retroverse (three out of four) or transverse (one out of four) and always deep. The metaloph is anterior to the hypocone in two out of four teeth and transversal in the remaining two. It is transversely directed in two and proverse in two specimens (Figure 6.10-11).

m1. The anterior cingulid is short (two out of three specimens) or long (one out of three). The metalophid I is well connected to the protoconid. In all the specimens the mesolophid is connected to the metaconid. The sinusid is M-shaped. The hypoconulid is present. The hypolophid is connected to the anterior arm of the hypoconid (Figure 6.12).

$\mathbf{m 2}$. The anterior cingulid is long. The metalophid I is well connected to the protoconid. The mesolophid is long and connected to the metaconid in one specimen and it is medium in the remaining one. The sinusid is M-shaped. The hypoconulid is pres- ent. In all specimens the hypolophid is connected to the anterior arm of the hypoconid (See Figure 6.12).

m3. The metalophid I is well connected to the protoconid. The mesolophid is short, the sinusid Mshaped. The hypoconulid is always present. The hypolophid is connected to the anterior arm of the hypoconid (Figure 6.12).

Remarks. Schmidt-Kittler et al. (2007) assigned all large-sized Yindirtemys specimens from Mongolia to $Y$. deflexus. Since new and rich fossil material from different localities is accessible to us, we are able to differ $Y$. suni from $Y$. deflexus.

Yindirtemys suni from the Mongolian localities differs from Y. deflexus by larger size of teeth, large and inflated jaws and bones. Upper molars with less tendency to develop additional crests (simpler pattern), and bulky and globular P4. In the lower molars, the protoconid and hypoconid are shorter than in Y. deflexus (see Appendix 1).

Yindirtemys birgeri Bendukidze, 1993. Figure 7.1-7.8.

1993 Yindirtemys birgeri Bendukidze, p. 64-65, 143.

1997 Yindirtemys sajakensis birgeri Bendukidze, p. 207. 2004 Yindirtemys birgeri Lopatin, p. 292-293.

2009 Yindirtemys birgeri Benduzidze, de Bruijn, and Van den Hoek Ostende, p. 351-352, 356, 368369.

Holotype. Palate with P4-M3 left and P4-M3 right. Bendukidze (1993: pl.XXI, fig. 2). 
Type Locality. Altyn Schokysu (Aral region, Kazakhstan).

Material and Measurements. Tatal Gol (TAT-051/ 2) NHMW 2012/0060/0001. Palate with P4-M3 left and P4-M3 right. See Table 5 and Appendix 3.

Stratigraphic Range in Mongolia. Upper part of the late Oligocene, Mongolian biozone C1; Hsanda Gol Fm.

Geographic Distribution. Valley of Lakes (Mongolia) and North Aral region (Kazakhstan).

Description. P4. The labial posteroloph is short (one of two specimens) or medium (one of two). The lingual posteroloph is connected to the protocone (two out of two). The lingual crest of the metaloph is long and connected to the posteroloph (two out of two).

M2. The two specimens are heavily worn and many of their morphological characters not visible. The labial anteroloph is connected to the paracone. The lingual anteroloph is absent.

M3. The labial anteroloph is long and connected to the paracone (one of two specimens) or it is medium long (one of two). The lingual anteroloph and the anterior groove are absent. The protoloph is anterior and transversely directed to the protocone. One specimen bears a deflexus structure and the other a deflexus structure and an anticrochet. The sinus is transverse and deep. The metaloph is connected transversely to the hypocone. The tooth morphology is swollen and bulky.

Remarks. The complete maxilla from Mongolia is similar in size to Yindirtemys birgeri Bendukidze, 1993 and Y. ambiguus Wang, 1997. However, both M3 from Mongolia have deflexus structure, and according to Wang, 1997, this structure is absent in Yindirtemys ambiguus. Tatal Gol (Mongolia) is the easternmost occurrence of Yindirtemys birgeri. So far this species has only been recorded from the Aral Fm. in Kazakhstan.

\section{RESULTS AND DISCUSSION}

The assemblages of Yindirtemys deflexus from the Valley of Lakes show a similar distribution of dental characters throughout the Mongolian biozone $\mathrm{C} 1$ (Appendix 1). The same situation is also valid for the Mongolian assemblages of $Y$. suni throughout biozones C1-D and D. However, both Yindirtemys deflexus and $Y$. suni increase in size through time (Figure 8). We tested the significance of the differences between the assemblages of Yindirtemys with an analysis of variance (One-Way ANOVA) and post hoc tests. The specimen numbers are too small $(<4)$ for statistical tests of $p 4$, $\mathrm{m} 1, \mathrm{dp} 4, \mathrm{M} 1$ and DP4. The statistical analyses of teeth (Table 6) show significant differences in the length of $\mathrm{m} 3$ between $Y$. deflexus from DEL-B/12 and RHN-A/7. These differences correspond to differences in the ages of the assemblages. DEL-B/ 12 is one of the oldest assemblages of the later Oligocene (biozone C1), whereas RHN-A/7 is one of the youngest of biozone $\mathrm{C} 1$. This points to a size increase of $\mathrm{m} 3$ within biozone $\mathrm{C} 1$. Nonetheless, despite of the size differences, the morphology remains rather constant among assemblages throughout biozone $\mathrm{C} 1$.

Our results agree with those of Benduzidze et al. (2009). Those authors suggest a trend towards a size increase in Yindirtemys ( $Y$. deflexus and $Y$. birgeri), more robust cusps, and a more forwards directed protoloph in the upper molars. We also recorded this trend in Yindirtemys suni (Figure 6).

At present, enough specimens are available for a comparison between $Y$. suni and $Y$. deflexus. Yindirtemys suni (from HTE-014-018) differs significantly in size from $Y$. deflexus (from DEL-B/12, TGW-A/surface, HTSE-009+013 and RHN-A/7 (Table 6 and Table 7) and the dental characters described before. Therefore, and contrary to Schmidt-Kittler et al. (2007), we consider Y. suni as a valid species and different from $Y$. deflexus.

\section{Biostratigraphic Issues}

The ctenodactylids are among the most abundant groups of rodent throughout the latest Oligocene (upper Chattian stage; Tabenbulukian Chinese Mammal Age) in Mongolia. Specifically, during the Mongolian biozone $\mathrm{C} 1$, Yindirtemys deflexus is one of the most abundant and characteristic species, serving as a "key fossil" for the latest Oligocene (biozone C1). Moreover, Yindirtemys suni is the "key fossil" for the Oligocene-Miocene transition (C1-D) and for the earliest Miocene biozone D (Aquitanian stage; Xieijan Chinese Mammal Age).

Yindirtemys birgeri co-occurs with Y. deflexus in the fauna of TAT-051/2 (biozone C1) (Figure 3). The type of Y. birgeri stems from Kazakhstan (Akotau) and the species is also represented in Altyn Schokysu (North Aral region in Kazakhstan). Bendukidze et al. (2009) revised the small mammals from the North Aral region and considered the age of the Aral Formation as Late Oligocene. Furthermore, they explain the differences between assemblages from the Aral Formation in Kazakhstan and the late Oligocene faunas of the Valley of Lakes to be ecological and not age differences. The occurrence of Castoridae in Kazakhstan (two genera and three species) indicates more humid environ- 

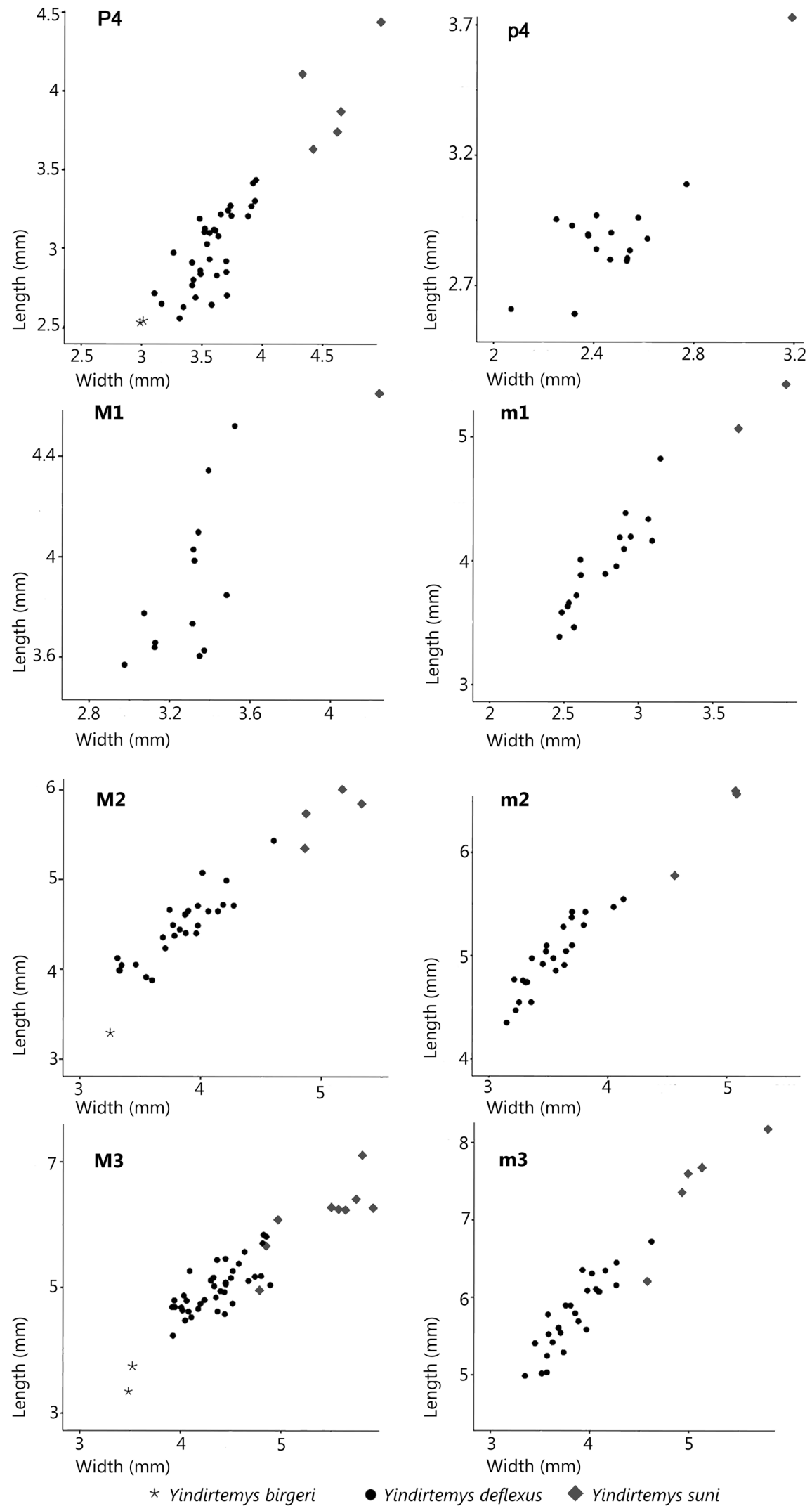

FIGURE 8. Distribution of the length and width ranges charts of the upper and lower dental elements for the three large-sized species of Yindirtemys from the Valley of Lakes. 
TABLE 6. Statistical analyses of the lengths of the cheek teeth of Yindirtemys deflexus and Y. suni. Abbreviations: N, number of specimens; df, degree of freedom; df2W, degree of freedom of the denominator corrected for Welch's test; $\mathrm{p}$, significance value; df2B-F, degree of freedom of the denominator corrected for Brown-Forsythe's test; $t$, T-student value; F, One-way ANOVA test. Significant differences in bold $(\alpha=0.05)$. All analyses use exclusively localities with sample sizes larger than four specimens.

\begin{tabular}{|c|c|c|c|c|c|c|c|c|c|c|c|c|}
\hline \multirow[b]{2}{*}{ Element } & \multirow[b]{2}{*}{$\mathbf{N}$} & \multirow{2}{*}{$\begin{array}{c}\text { Y. deflexus } \\
\text { Locality }\end{array}$} & \multirow{2}{*}{$\begin{array}{l}\text { Y. suni } \\
\mathbf{y}\end{array}$} & \multicolumn{3}{|c|}{ Welch's test } & \multicolumn{2}{|c|}{$\begin{array}{c}\text { Brown- } \\
\text { Forsythe's test }\end{array}$} & \multicolumn{2}{|c|}{ Student's test } & \multicolumn{2}{|c|}{ ANOVA } \\
\hline & & & & df & df2W & $\mathbf{p}$ & df2B-F & $\mathbf{p}$ & $t$ & $p$ & $F$ & $p$ \\
\hline \multirow[t]{2}{*}{$\mathrm{m} 1$} & 4 & RHN-023 & & 8 & & & & & 0.137 & 0.723 & & \\
\hline & 5 & RHN-A/7 & & & & & & & & & & \\
\hline \multirow[t]{3}{*}{$\mathrm{m} 2$} & 4 & $\mathrm{RHN}-023$ & & 15 & & & & & & & 0.046 & 0.956 \\
\hline & 8 & RHN-A/7 & & & & & & & & & & \\
\hline & 4 & DEL-B/12 & & & & & & & & & & \\
\hline \multirow[t]{2}{*}{ m3 } & 6 & RHN-A/7 & & 17 & & & & & 31.723 & 0.000 & & \\
\hline & 12 & DEL-B/12 & & & & & & & & & & \\
\hline \multirow[t]{3}{*}{$\mathrm{P} 4$} & 6 & HTSE-009+013 & & & & & & & & & & \\
\hline & 9 & RHN-A/7 & & 19 & & & & & & & 2.914 & 0.082 \\
\hline & 5 & TGW-A/surface & & & & & & & & & & \\
\hline \multirow[t]{5}{*}{ M2 } & 4 & HTSE-009+013 & & 4 & 8.230 & & & 0.428 & & & & \\
\hline & 5 & RHN-023 & & & & & & & & & & \\
\hline & 4 & TGW-A/5 & & & & 0.280 & 9.854 & & & & & \\
\hline & 5 & TGW-A/surface & & & & & & & & & & \\
\hline & 4 & DEL-B/12 & & & & & & & & & & \\
\hline \multirow[t]{5}{*}{ M3 } & 4 & & HTE-14-18 & 27 & & & & & & & 14.826 & 0.000 \\
\hline & 9 & HTSE-009+013 & & & & & & & & & & \\
\hline & 6 & RHN-A/7 & & & & & & & & & & \\
\hline & 4 & TGW-A/surface & & & & & & & & & & \\
\hline & 5 & DEL-B/12 & & & & & & & & & & \\
\hline
\end{tabular}

ments than in Mongolia. The co-occurrence of $Y$. birgeri and Y. deflexus in Mongolia and Kazakhstan supports the hypothesis of similar ages for both localities.

\section{Paleobiogeographic Considerations and Paleoecological Implications}

The Yindirtemys species can be divided into three size groups: 1) The small-sized species $Y$. bohlini (Huang, 1985), Y. grangeri (Bohlin, 1946) Y. shevyrevae Vianey-Liaud et al., 2006 and Y. ulantatalensis (Huang, 1985); 2) The medium-sized species Y. birgeri, Y. ambiguus Wang, 1997 and Y. xiningensis Wang, 1997; and 3) The larger-sized species $Y$. deflexus and $Y$. suni. We follow Schmidt-Kitter et al. (2007) and consider Y. gobiensis Kowalski, 1974 a synonym of Y. deflexus.

The small Yindirtemys ulantatalensis is recorded from the Ulantatal area and Saint Jacques (Inner Mongolia) and occurred from the early to late Oligocene (Vianey-Liaud et al., 2006).
It was also recognized in the late Oligocene (biozone C) of Mongolia (Schmidt-Kittler et al., 2007). Beyond that, $Y$. shevyrevae occurred earlier in Mongolia (early Oligocene, biozone B) than in China (late Oligocene). The other two small-sized species, $Y$. bohlini and $Y$. grangeri, are restricted to the late Oligocene of China. Yindirtemys bohlini from Inner Mongolia (Ulantatal area and Saint Jacques) and Y. grangeri (Gansu and Quanlishan provinces) are endemic species of China.

To date, the medium-sized Yindirtemys birgeri was known only from Kazakhstan (Bendukidze et al., 2009), and is now described from Mongolia. All other medium-sized species are known only from China. Yindirtemys ambiguus occurred during the late Oligocene and $Y$. xiningensis during the early Miocene. Yindirtemys ambiguus resembles $Y$. birgeri and differs only by the lack of the "deflexus" structure of the M3 and by the more posteriorly directed metalophs of upper molars (Wang, 1997). 
TABLE 7. Post hoc (Tukey's-b) of the M3 of Yindirtemys deflexus and Y. suni. Significant differences are indicated in bold. The mean difference is significant at the 0.05 level.

\begin{tabular}{|c|c|c|c|}
\hline & Localities & & Mean difference \\
\hline \multirow{20}{*}{ 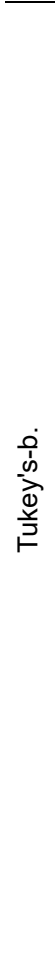 } & & HTE-14-18 & -1.664 \\
\hline & DEL-B/12 & HTSE-009+013 & -0.263 \\
\hline & Y. deflexus & $\mathrm{RHN}-\mathrm{A} / 7$ & -0.416 \\
\hline & & TGW-A/surface & 0.07525 \\
\hline & & DEL-B/12 & 1.664 \\
\hline & HTE-14-18 & HTSE-009+013 & 1.401 \\
\hline & Y. suni & RHN-A/7 & 1,248 \\
\hline & & TGW-A/surface & 1.73925 \\
\hline & & DEL-B/12 & 0.263 \\
\hline & HTSE-009+013 & HTE-14-18 & -1.401 \\
\hline & Y. deflexus & RHN-A/7 & -0.153 \\
\hline & & TGW-A/surface & 0.33825 \\
\hline & & DEL-B/12 & 0.416 \\
\hline & RHN-A/7 & HTE-14-18 & -1.248 \\
\hline & Y. deflexus & HTSE-009+013 & 0.153 \\
\hline & & TGW-A/surface & 0.49125 \\
\hline & & DEL-B/12 & -0.07525 \\
\hline & TGW-A/surface & HTE-14-18 & -1.73925 \\
\hline & Y. deflexus & HTSE-009+013 & -0.33825 \\
\hline & & RHN-A/7 & -0.49125 \\
\hline
\end{tabular}

The large-sized Yindirtemys deflexus has the widest distribution: from the Gansu province and Inner Mongolia (China), to the Valley of Lakes (Mongolia), and as far as the Aral region (Kazakhstan) in the west. The largest species is $Y$. suni. It occurred in the Qinghai and Gansu provinces and in Inner Mongolia (China), and we describe it in detail from the Valley of Lakes (Mongolia).

The distribution of the Ctenodactylidae shows that no major geographical barriers prevented mammal movement between Kazakhstan, Mongolia, and northern China throughout the Oligocene. In this area the differences of isochronous mammal communities apparently derive from locally different environments and/or climatic conditions and from the development of ecological niches (Schmidt-Kittler et al., 2007; Bendukidze et al., 2009; Gomes Rodrigues et al., 2014).

Our studies on Yindirtemys teeth from Mongolia demonstrate trends towards increasing size, crown height, and more developed crests. These modifications started after the heyday of Ctenodacylidae in the late Oligocene (biozone C), increased rapidly towards the latest Oligocene (biozone C1), and ended with the extinction of the genus in the early Miocene (biozone D). Not only
Yindirtemys but the entire subfamily Tataromyinae diversified and flourished during the Oligocene, spread from East to Central Asia, and became extinct in the earliest Miocene.

Based on sedimentological evidence, we interpret the history of Yindirtemys in Asia as a response to paleoenvironmental changes caused by paleogeographic reorganisations and by climate changes towards increasing aridity and widespread desert formation (Guo et al., 2002, 2008; Sun et al., 2010; Harzhauser et al., 2016).

\section{CONCLUSIONS}

The Yindirtemys fossils from the Valley of Lakes (Mongolia) provide insights into the evolution, biostratigraphy and paleobiogeography of the genus in Asia. During the latest Oligocene and earliest Miocene, three species occurred: Y. deflexus, $Y$. birgeri and $Y$. suni. The most abundant species, $Y$. deflexus is restricted to the latest Oligocene (biozones $\mathrm{C} 1$ and $\mathrm{C} 1-\mathrm{D}$ ). It spreads through Kazakhstan, Mongolia, Inner Mongolia and North China. Yindirtemys birgeri co-occurred with $Y$. deflexus in the Tatal Gol assemblage TAT-051/2 (biozone C1). Geographically it ranges from Kazakhstan to Mongolia. Yindirtemys birgeri is 
described here for the first time in Mongolia. The largest species, Y. suni, is recorded from the Oligocene-Miocene transition (biozone C1-D) and from the earliest Miocene (biozone D), and ranges from Mongolia to China. All three species follow the same trend of dental pattern: a stable dental morphology with increase in size.

The genus Yindirtemys was very abundant and diverse in Asia. Six small-sized species, three medium-sized and two large-sized species occurred during the Oligocene and early Miocene in China, Mongolia and Kazakhstan. This suggests that no physical barriers existed between these countries.

\section{ACKNOWLEDGMENTS}

We thank for their support all Mongolian and European contributors to the fieldwork, administration and scientific work. We are grateful to our Mongolian Project partners D. Badamgarav, R. Barsbold, Yo. Khand and B. Bayarmaa. Y. Radnaa and the nomad families Ch. Tomurbaatar and L. Olzibaatar participated in the fieldwork. Our nomadic friends organized the field camp and food supply, and supported the sampling and screening process for many years. We would like to thank the two anonymous referees for their valuable comments on the manuscript. We thank E. Höck, who took the SEM-images and established an image database of small mammal fossils from Mongolia. We acknowledge U. Göhlich (NHMW) for the facilities to study the collections and M. Stachowitsch for improving the English. Our research was granted by several projects of the Austrian Science Fund (P-10505-GEO, P-15724-N06 and P-23061N19). A. Oliver was supported by a project research contract (FWF: P-23061-N19).

\section{REFERENCES}

Bendukidze, O.G. 1993. Melkie mlekopitayushchie miotsena Yugo-Zapadnogo Kazakhstana i Turgaya. Small mammal of Miocene from south-western Kazakhstan and Turgai. Unpublished Ph.D. Thesis, Tbilisi University, Metsniereba, Kazakhstan.

Bendukidze, O.G. 1997. The Oligocene rodents of central and western Kazakhstan and their stratigraphic significance. Memoires et Travaux de I'Institut de Montpellier de l'Ecole Pratique des Hautes Etudes, 21:205-208.

Bendukidze, O.G., de Bruijn, H., and Van den Hoek Ostende, L.W. 2009. A revision of Late Oligocene associations of small mammals from the Aral Formation (Kazakhstan) in the National Museum of Georgia, Tbilissi. Palaeodiversity, 2:343-377.
Bohlin, B. 1946. The fossil Mammals from the Tertiary deposit of Taben-buluk, Western Kansu. Part II: Simplicidentata, Carnivora, Artiodactyla, Perissodactyla, and Primates. Palaeontologia Sinica 8b:1-259.

Bowdich, T.E. 1821. An Analysis of the Natural Classification of Mammalia for the Use of Students and Travellers. Smith, J., Paris.

Daxner-Höck, G. and Badamgarav, D. 2007. OligoceneMiocene Vertebrates from the Valley of Lakes (Central Mongolia): Morphology, phylogenetic and stratigraphic implications - 1. Geological and stratigraphic setting. Annalen des Naturhistorischen Museums in Wien, 108A:1-24.

Daxner-Höck, G., Badamgarav, D., and Erbajeva, M. 2010. Oligocene Stratigraphy Based on a SedimentBasalt Association in Central Mongolia (Taatsiin Gol and Taatsiin Tsagaan Nuur Area, Valley of Lakes): Review of a Mongolian-Austrian Project. Vertebrata Palasiatica, 48(4):348-366.

Daxner-Höck, G., Badamgarav, D., and Maridet, O. 2014. Dipodidae (Rodentia, Mammalia) from the Oligocene and Early Miocene of Mongolia. Annalen des Naturhistorischen Museums in Wien, 116:131-214.

Daxner-Höck, G., Badamgarav, D., and Maridet, $O$. 2015. Evolution of Tachyoryctoidinae (Rodentia, Mammalia): evidences of the Oligocene and Early Miocene of Mongolia. Annalen des Naturhistorischen Museums in Wien, 117:161-195.

Daxner-Höck, G., Höck, V., Badamgarav, D., Furtmüller, G., Frank, W., Montag, O., and Schmid, H. 1997. Cenozoic stratigraphy based on a sediment-basalt association in Central Mongolia as requirement for correlation across Central Asia. Mémoires et travaux de I'Institut de Montpellier 21:163-176.

Gomes Rodrigues, H., Marivaux, L., and Vianey-Liaud, M. 2012. Expansion of open landscapes in Northern China during the Oligocene induced by dramatic climate changes: Paleoecological evidence. Palaeogeography, Palaeoclimatology, Palaeoecology, 358:6271.

Gomes Rodrigues, H., Marivaux, L., and Vianey-Liaud, M. 2014. Rodent paleocommunities from the Oligocene of Ulantatal (Inner Mongolia, China). Paleovertebrata, 38(1):1-11.

Gradstein, F.M., Ogg, J.G., and Smith, A.G. 2004. A Geologic Time Scale 2004. Cambridge University Press, Cambridge.

Guo, Z.T., Ruddiman, W.F., Hao, Q.Z., Wu, H.B., Qiao, Y.S., Zhu, R.X., Peng, S.Z., Wei, J.J., Yuan, B.Y., and Liu, T.S. 2002. Onset of Asian desertification by 22 Myr ago inferred from loess deposits in China. Nature, 416:159-163.

Guo, Z.T., Sun, B., Zhang, Z.S., Peng, S.Z., Xiao, G.Q., Ge, J.Y., Hao, Q.Z., Qiao, Y.S., Liang, M.Y., Liu, J.F., Yin, Q.Z., and Wei, J.J. 2008. A major reorganization of Asian climate by the early Miocene. Climate of the Past, 4:153-174.

Harzhauser, M., Daxner-Höck, G., López-Guerrero, P., Maridet, O., Oliver, A., Piller, W.E., Richoz, S., Erba- 
jeva, M.A., and Göhlich, U.B. 2016. The stepwise onset of the Icehouse world and its impact on Oligocene-Miocene Central Asian mammal communities. Scientific Reports, 6:36169, doi: 10.1038/srep36169.

Höck, V., Daxner-Höck, G., Schmid, H.P., Badamgarav, D., Frank, W., Furtmuller, G., Montag, O., Barsbold, R., Khand, Y., and Sodov, J. 1999. Oligocene-Miocene sediments, fossils and basalts from the Valley of Lakes (Central Mongolia) - an integrated study. Mitteilungen der Oesterreichischen Geologischen Gesellschaft, 90:83-125.

Huang, X.S. 1985. Middle Oligocene Ctenodactylids (Rodentia, Mammalia) of Ulantatal, Nei Mongol. Vertebrata Palasiatica, 23(1):27-37.

Kowalski, K. 1974. Middle Oligocene rodents from Mongolia. Palaeontologia Polonica, 30:147-178.

Lavocat, R. 1961. Etude systématique de la faune de mammifères et conclusions générales. Notes et Mémoires du Service géologique du Maroc, 115:109142.

Li, C.K. and Qiu, Z.D. 1980. Early Miocene mammalian fossils of Xining-Basin, Qinghai. Vertebrata Palasiatica, 18(3):198-215.

Lopatin, A.V. 2004. Early Miocene small mammals from the North Aral Region (Kazakhstan) with special reference to their biostratigraphic significance - Introduction. Paleontological Journal, 38:S217-S323.

Mellett, J.S. 1968. The Oligocene Hsanda Gol formation, Mongolia: a revised fauna list. American Museum Novitates, 2318:1-16.

Meng, J., Ye, J., Wu, W.-Y., Yue, L.-P., and Ni, X.-J. 2006. A recommended boundary stratotype section for Xiejian stage from northern Junggar basin: implications to related bio-chronostratigraphy and environmental changes. Vertebrata Palasiatica, 44(3):205-236.

Qiu, Z. and Gu, Z. 1988. A new locality yielding Mid-Tertiary mammals near Lanzhou, Gansu. Vertebrata Palasiatica, 26(3):198-213.

Schaub, S. 1958. Simplicidentata (= Rodentia). Traité de paléontologie, 6(2):659-818.

Schmidt-Kittler, N., Vianey-Liaud, M., and Marivaux, L. 2007. Oligocene-Miocene Vertebrates from the Val- ley of Lakes (Central Mongolia): Morphology, phylogenetic and stratigraphic implications - 6. The Ctenodactylidae (Rodentia, Mammalia). Annalen des Naturhistorischen Museums in Wien, 108A:173-215.

SPSS 2006. Statistical Package for the Social Sciences for Windows. SPSS Inc., Chicago.

Stehlin, H.G. and Schaub, S. 1951. Die Trigonodontie der Simplicidentaten Nager. Schweizerische Palaeontologische Abhandlungen, 67(1950):2-385.

Sun, J., Ye, J., Wu, W., Ni, X., Bi, S., Zhang, Z., Liu, W., and Meng, J. 2010. Late Oligocene-Miocene mid-latitude aridification and wind patterns in the Asian interior. Geology, 38:515-518.

Teilhard de Chardin, P. 1926. Description de Mammiferes tertiaires de Chine et de Mongolie. Annales de Paleontologie, 15:1-52.

Teilhard de Chardin, P. and Leroy, P. 1942. Chinese fossil mammals. A complete bibliography analysed, tabulated, annotated and indexed. Institut de GéoBiologie Pékin 8:1-142.

Vianey-Liaud, M., Schmidt-Kittler, N., and Marivaux, L. 2006. The Ctenodactylidae (Rodentia) from the Oligocene of Ulantatal (Inner Mongolia, China). Paleovertebrata, 34(3-4):111-206.

Vianey-Liaud, M., Rodrigues, H.G., and Marivaux, L. 2010. A new Oligocene Ctenodactylinae (Rodentia: Mammalia) from Ulantatal (nei Mongol): new insight on the phylogenetic origins of the modern Ctenodactylidae. Zoological Journal of the Linnean Society, 160(3):531-550.

Wang, B. 1994. The Ctenodactyloidea of Asia. National Science Museum Monographs, 8:35-47.

Wang, B. 1997. The Mid-Tertiary Ctenodactylidae (Rodentia, Mammalia) of eastern and central Asia. Bulletin of the American Museum of Natural History, 234:1-88.

Wang, B.Y., Chang, J.A., Meng, X.I., and Chen, J.R. 1981.Stratigraphy of the upper and middle Oligocene of Qianlishan district, Nei Mongol (Inner Mongolia). Vertebrata Palasiatica, 19(1):26-34.

Zittel, K. 1893. Traite de Paleontologie III, Paleozoologie Vertebrata (Pisces, Amphibia, Reptilia, Aves). Paris. 


\section{APPENDIX 1.}

Distribution of character states (available online as PDF download at palaeo-electronica.org/content/2017/1729-ctenodactylidae-from-mongolia).

\section{APPENDIX 2.}

Description of the character states (available online as PDF download at palaeo-electronica.org/ content/2017/1729-ctenodactylidae-from-mongolia). 


\section{APPENDIX 3.}

Three dimensional interactive figure of Yindirtemys birgeri from the locality of Tatal Gol (TAT-051/ 2), Valley of Lakes (Mongolia). Palate with P4-M3 left and P4-M3 right. NHMW 2012/0060/000 (available online as PDF download at palaeo-electronica.org/content/2017/1729-ctenodactylidae-from-mongolia).

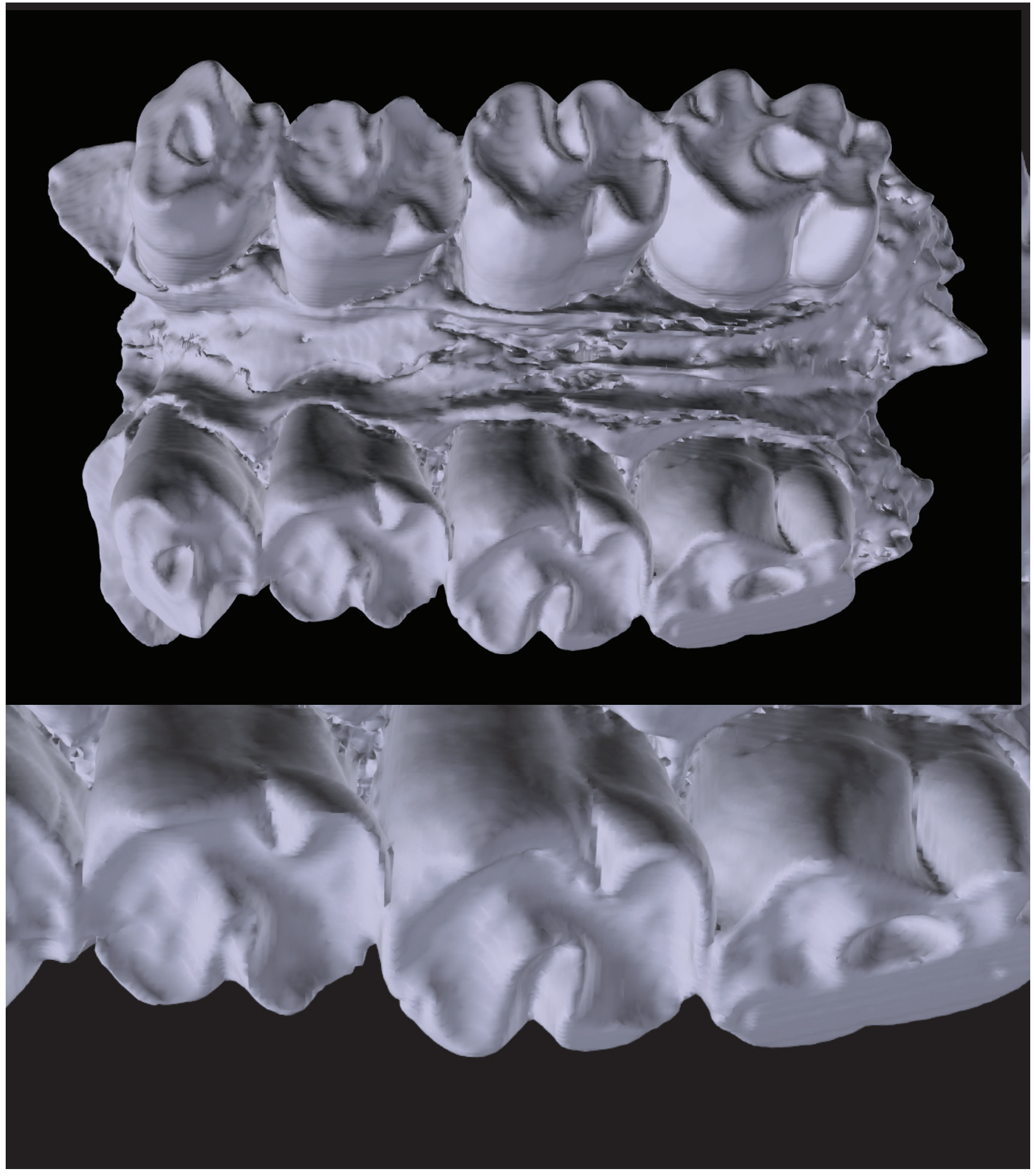

